\title{
Pacific
}

Journal of

Mathematics

\section{CLOSED ORBITS OF A CHARGE IN A WEAKLY EXACT MAGNETIC FIELD}

WILL J. MERRY 


\title{
CLOSED ORBITS OF A CHARGE IN A WEAKLY EXACT MAGNETIC FIELD
}

\author{
WILL J. MERRY
}

\begin{abstract}
We prove that for a weakly exact magnetic system on a closed connected Riemannian manifold, almost all energy levels contain a closed orbit. More precisely, we prove the following stronger statements. Let $(M, g)$ denote a closed connected Riemannian manifold and $\sigma \in \Omega^{2}(M)$ a weakly exact 2form. Let $\phi_{t}: T M \rightarrow T M$ denote the magnetic flow determined by $\sigma$, and let $c(g, \sigma) \in \mathbb{R} \cup\{\infty\}$ denote the Mañé critical value of the pair $(g, \sigma)$. We prove that if $k>c(g, \sigma)$, then for every nontrivial free homotopy class of loops on $M$ there exists a closed orbit of $\phi_{t}$ with energy $k$ whose projection to $M$ belongs to that free homotopy class. We also prove that for almost all $k<c(g, \sigma)$ there exists a closed orbit of $\phi_{t}$ with energy $k$ whose projection to $M$ is contractible. In particular, when $c(g, \sigma)=\infty$ this implies that almost every energy level has a contractible closed orbit. As a corollary we deduce that a weakly exact magnetic flow with $[\sigma] \neq 0$ on a manifold with amenable fundamental group (which implies $c(g, \sigma)=\infty$ ) has contractible closed orbits on almost every energy level.
\end{abstract}

\section{Introduction}

Let $(M, g)$ denote a closed connected $d$-dimensional Riemannian manifold, with tangent bundle $\pi: T M \rightarrow M$ and universal cover $\tilde{M}$. We will assume $M$ is not simply connected, as otherwise $\widetilde{M}=M$ and all results proved in this paper reduce to special cases of the results in [Contreras 2006]. Let $\sigma \in \Omega^{2}(M)$ denote a closed 2-form, and let $\tilde{\sigma} \in \Omega^{2}(\tilde{M})$ denote its pullback to the universal cover. In this paper we consider the case where $\sigma$ is weakly exact, that is, when $\tilde{\sigma}$ is exact (this is equivalent to requiring that $\left.\sigma\right|_{\pi_{2}(M)}=0$ ); however we do not assume that $\tilde{\sigma}$ necessarily admits a bounded primitive.

Let $\omega_{g}$ denote the standard symplectic form on $T M$ obtained by pulling back the canonical symplectic form $d q \wedge d p$ on $T^{*} M$ via the Riemannian metric. Let $\omega:=$ $\omega_{g}+\pi^{*} \sigma$ denote the twisted symplectic form determined by the pair $(g, \sigma)$. Let $E: T M \rightarrow \mathbb{R}$ denote the energy Hamiltonian $E(q, v)=\frac{1}{2}|v|^{2}$. Let $\phi_{t}: T M \rightarrow T M$

MSC2000: 37J45, 70H12.

Keywords: magnetic flow, twisted geodesic flow, periodic orbits, Mañé critical value. 
denote the flow of the symplectic gradient of $E$ with respect to $\omega$; such $\phi_{t}$ is known as a twisted geodesic flow or a magnetic flow. The reason for the latter terminology is that this flow can be thought of as modeling the motion of a particle of unit mass and unit charge under the effect of a magnetic field represented by the 2-form $\sigma$. Given $k \in \mathbb{R}^{+}:=\{t \in \mathbb{R}: t>0\}$, let $\Sigma_{k}:=E^{-1}(k) \subseteq T M$.

There exists a number $c=c(g, \sigma) \in \mathbb{R} \cup\{\infty\}$, the Mañé critical value (see [Mañé 1996; Contreras et al. 1997; Contreras and Iturriaga 1999; Burns and Paternain 2002] or Section 2 for the precise definition), such that the dynamics of the hypersurface $\Sigma_{k}$ differs dramatically depending on whether $k<c, k=c$ or $k>c$. Moreover $c<\infty$ if and only if $\tilde{\sigma}$ admits a bounded primitive.

In this paper we study the old problem of the existence of closed orbits on prescribed energy levels. In the case when $\sigma$ is exact, this has been essentially solved by Contreras [2006]; see Theorem D therein in particular, which gives contractible closed orbits in almost every energy level below the Mañé critical value, and closed orbits in every free homotopy class for any energy level above the critical value. In the case of surfaces a stronger result is known to hold: Contreras, Macarini and Paternain have proved in [Contreras et al. 2004, Theorem 1.1] that in this case every energy level admits a closed orbit. However the case of a magnetic monopole (that is, when $\sigma$ is not exact) remains open, although much progress has been made. Let us describe now some of these results. A more comprehensive survey can be found in the introduction to [Contreras et al. 2004]; see also [Ginzburg 1996] for a introductory account of the problem.

Macarini [2004], extending an earlier result of Polterovich [1998], proved that if $[\sigma] \neq 0$ there exist nontrivial contractible closed orbits of the magnetic flow in a sequence of arbitrarily small energy levels. Kerman [2000] proved the same result for magnetic fields given by symplectic forms. This was then sharpened by Ginzburg and Gürel [2009] and finally by Usher [2009], where it is proved that when $\sigma$ is symplectic, contractible closed orbits exist for all low energy levels. See also [Lu 2006] for another interesting approach to the problem in the case of symplectic $\sigma$. Perhaps the most general result so far is due to Schlenk [2006], who showed that for any closed 2-form (not necessarily weakly exact), almost every sufficiently small energy level contains a contractible closed orbit.

This paper extends [Contreras 2006, Theorem D] to the weakly exact case.

Theorem 1.1. Let $(M, g)$ denote a closed connected Riemannian manifold, and let $\sigma \in \Omega^{2}(M)$ denote a closed 2-form whose pullback to the universal cover $\widetilde{M}$ is exact. Let $c=c(g, \sigma) \in \mathbb{R} \cup\{\infty\}$ denote the Mañé critical value, and let $\phi_{t}$ denote the magnetic flow defined by $\sigma$.

(1) If $c<\infty$, then for all $k>c$ and for each nontrivial homotopy class $v \in[\mathbb{\mathbb { T }}, M]$, there is a closed orbit of $\phi_{t}$ with energy $k$ whose projection to $M$ belongs to $v$. 
(2) For almost all $k \in(0, c)$, where possibly $c=\infty$, there exists a contractible closed orbit of $\phi_{t}$ with energy $k$.

Theorem 1.1(1) has, under a mild technical assumption on $\pi_{1}(M)$, been proved by Paternain [2006]. We use a completely different method of proof however, which bypasses the need for this additional assumption. For $c(g, \sigma)<\infty$, Theorem 1.1(2) is due to Osuna [2005]; we believe the main contribution of this paper is the case $c(g, \sigma)=\infty$.

Remark. We will actually prove a slightly stronger statement than the one stated above; see Proposition 5.8 below for details.

When $\pi_{1}(M)$ is amenable and $\sigma$ is not exact, we always have $c(g, \sigma)=\infty$; see for instance [Paternain 2006, Corollary 5.4]. Thus the following corollary is immediate.

Corollary 1.2. Let $(M, g)$ denote a closed connected Riemannian manifold, and let $\sigma \in \Omega^{2}(M)$ denote a closed nonexact 2-form whose pullback to the universal cover $\widetilde{M}$ is exact. Suppose $\pi_{1}(M)$ is amenable. Then almost every energy level contains a contractible closed orbit of the magnetic flow defined by $\sigma$.

Let us now give a brief outline of our method of attack. Fix a primitive $\theta$ of $\tilde{\sigma}$, and consider the Lagrangian $L: T \widetilde{M} \rightarrow \mathbb{R}$ defined by

$$
L(q, v):=\frac{1}{2}|v|^{2}-\theta_{q}(v) .
$$

The Euler-Lagrange flow of $L$ is precisely the lifted flow $\tilde{\phi}_{t}: T \widetilde{M} \rightarrow T \widetilde{M}$ of the magnetic flow $\phi_{t}: T M \rightarrow T M$; see for example [Contreras and Iturriaga 1999]. Recall that the action $A(y)$ of the Lagrangian $L$ over an absolutely continuous curve $y:[0, T] \rightarrow \widetilde{M}$ is given by

$$
A(y):=\int_{0}^{T} L(y(t), \dot{y}(t)) d t=\int_{0}^{T} \frac{1}{2}|\dot{y}(t)|^{2} d t-\int_{y} \theta .
$$

Set

$$
A_{k}(y):=\int_{0}^{T}(L(y(t), \dot{y}(t))+k) d t=A(y)+k T .
$$

A closed orbit of $\tilde{\phi}_{t}$ with energy $k$ can be realized as a critical point of the functional $y \mapsto A_{k}(y)$. More precisely, let $\Lambda_{\tilde{M}}$ denote the Hilbert manifold of absolutely continuous square integrable curves $x: \mathbb{T} \rightarrow \widetilde{M}$ and consider $\tilde{S}_{k}: \Lambda_{\widetilde{M}} \times \mathbb{R}^{+} \rightarrow \mathbb{R}$ defined by

$$
\tilde{S}_{k}(x, T):=\int_{0}^{1} T \cdot L(x(t), \dot{x}(t) / T) d t+k T=\int_{0}^{1} \frac{1}{2 T}|\dot{x}(t)|^{2} d t+k T-\int_{x} \theta .
$$

Then the pair $(x, T)$ is a critical point $\tilde{S}_{k}$ if and only if $y(t):=x(t / T)$ is the projection to $\widetilde{M}$ of a closed orbit of $\tilde{\phi}_{t}$ with energy $k$; see [Contreras et al. 2000]. 
If $\sigma$ was actually exact then we could define $L$ on $T M$, instead of just on $T \widetilde{M}$. In this case it has been shown in [Contreras et al. 2000] that $\tilde{S}_{k}$ for $k>c(g, \sigma)$ satisfies the Palais-Smale condition and is bounded below. Standard results from Morse theory [Contreras et al. 2000, Corollary 23] then tell us that $\tilde{S}_{k}$ admits a global minimum, and this gives us our desired closed orbit. In [Contreras 2006] this was extended to give contractible orbits on almost every energy level below the critical value. Crucially however, these results use compactness of $M$ and hence are not applicable directly in the weakly exact case, since then $L$ is defined only on $T \widetilde{M}$.

In the weakly exact case, whilst $\tilde{S}_{k}$ is not well defined on $T M$, its differential is. This leads to our key observation that we can still work directly on $\Lambda_{M}$. More precisely, we define a functional $S_{k}: \Lambda_{M} \times \mathbb{R}^{+} \rightarrow \mathbb{R}$ with $^{1}$ the property that $(x, T)$ is a critical point of $S_{k}$ if and only if a lift $\tilde{y}$ to $\tilde{M}$ of the curve $y(t):=x(t / T)$ is the projection to $\widetilde{M}$ of a flow line of $\tilde{\phi}_{t}$ with energy $k$. The functional $S_{k}$ is given by

$$
S_{k}(x, T):=\int_{0}^{1} \frac{1}{2 T}|\dot{x}(t)|^{2} d t+k T-\int_{C(x)} \sigma,
$$

where $C(x)$ is any cylinder with boundary $x(\mathbb{T}) \cup x_{v}(\mathbb{T})$, where $x_{v} \in \Lambda_{M}$ is some fixed reference loop in the free homotopy class $v \in[\mathbb{T}, M]$ that $x$ belongs to. If $c(g, \sigma)<\infty$, then since $\sigma$ is weakly exact, the value $\int_{C(x)} \sigma$ is independent of the choice of cylinder $C(x)$ for any curve $x \in \Lambda_{M}$. In the case $c(g, \sigma)=\infty$, the value $\int_{C(x)} \sigma$ is independent of the choice of cylinder only when $x$ is a contractible loop.

The functional $S_{k}$ allows one to extend other results previously known only for the exact case to the weakly exact case. For instance, in [Merry 2010] we will use $S_{k}$ to establish the short exact sequence [Cieliebak et al. 2010; Abbondandolo and Schwarz 2009a] between the Rabinowitz Floer homology of a weakly exact twisted cotangent bundle and the singular (co)homology of the free loop space.

\section{Preliminaries}

The setup. It will be convenient to view $M$ and $\tilde{M}$ as being embedded isometrically in some $\mathbb{R}^{N}$ (which is possible by Nash's theorem). We will be interested in various spaces of absolutely continuous curves.

Given $q_{0}, q_{1} \in M$ and $T \geq 0$, let $C_{M}^{\text {ac }}\left(q_{0}, q_{1} ; T\right)$ denote the set of absolutely continuous curves $y:[0, T] \rightarrow M$ with $y(0)=q_{0}$ and $y(T)=q_{1}$. Let

$$
C_{M}^{\mathrm{ac}}\left(q_{0}, q_{1}\right):=\bigcup_{T \geq 0} C_{M}^{\mathrm{ac}}\left(q_{0}, q_{1} ; T\right)
$$

${ }^{1}$ If $\tilde{\sigma}$ does not admit any bounded primitives, $S_{k}$ is only defined on $\Lambda_{0} \times \mathbb{R}^{+}$, where $\Lambda_{0} \subseteq \Lambda_{M}$ is the subset of contractible loops. 
We can repeat the construction on $\widetilde{M}$ to obtain for $q_{0}, q_{1} \in \widetilde{M}$ sets $C_{\widetilde{M}}^{\text {ac }}\left(q_{0}, q_{1} ; T\right)$ and $C_{\widetilde{M}}^{\text {ac }}\left(q_{0}, q_{1}\right)$ of curves on $\widetilde{M}$.

Next, consider the space $W^{1,2}\left(\mathbb{R}^{N}\right)$ of absolutely continuous maps $x: I \rightarrow \mathbb{R}^{N}$ such that $\int_{0}^{1}|\dot{x}(t)|^{2} d t<\infty$ and the space

$$
W^{1,2}(M):=\left\{x \in W^{1,2}\left(\mathbb{R}^{N}\right): x(I) \subseteq M\right\},
$$

with $W^{1,2}(\tilde{M})$ defined similarly. Here and throughout, $I:=[0,1]$.

Let $\Lambda_{\mathbb{R}^{N}} \subseteq W^{1,2}\left(\mathbb{R}^{N}\right)$ denote the set of closed loops of class $W^{1,2}$ on $\mathbb{R}^{N}$, and let $\Lambda_{M}:=W^{1,2}(M) \cap \Lambda_{\mathbb{R}^{N}}$. We will think of maps $x \in \Lambda_{M}$ as maps $x: \mathbb{T} \rightarrow M$ (here $\mathbb{T}=\mathbb{R} / \mathbb{Z}$, which we shall often identify with $S^{1}$ ). Given a free homotopy class $v \in[\mathbb{T}, M]$, let $\Lambda_{v} \subseteq \Lambda_{M}$ denote the connected component of $\Lambda_{M}$ consisting of the loops belonging to $\nu$.

The tangent space to $\Lambda_{\mathbb{R}^{N}}$ at $x \in \Lambda_{\mathbb{R}^{N}}$ is given by

$$
T_{x} \Lambda_{\mathbb{R}^{N}}=\left\{\xi \in W^{1,2}\left(\mathbb{R}^{N}\right): \xi(0)=\xi(1)\right\} .
$$

Given $(x, T) \in \Lambda_{M} \times \mathbb{R}^{+}$, we thus have

$$
T_{(x, T)}\left(\Lambda_{M} \times \mathbb{R}^{+}\right)=\left\{(\xi, \psi) \in W^{1,2}\left(\mathbb{R}^{N}\right) \times \mathbb{R}: \xi(0)=\xi(1)\right\} .
$$

Let $\langle\cdot, \cdot\rangle$ denote the standard Euclidean metric. The metric on $W^{1,2}\left(\mathbb{R}^{N}\right)$ we will work with is

$$
\langle\xi, \zeta\rangle_{1,2}:=\langle\xi(0), \zeta(0)\rangle+\int_{0}^{1}\langle\dot{\xi}(t), \dot{\zeta}(t)\rangle d t
$$

This defines a metric that we shall denote simply by $\langle\cdot, \cdot\rangle$ on $W^{1,2}\left(\mathbb{R}^{N}\right) \times \mathbb{R}^{+}$by

$$
\langle(\xi, \psi),(\zeta, \chi)\rangle:=\langle\xi, \zeta\rangle_{1,2}+\psi \chi \text {. }
$$

Mañés critical value. We now recall the definition of $c(g, \sigma)$, the critical value introduced in [Mañé 1996], which plays a decisive role in all that follows.

Let us fix a primitive $\theta$ of $\tilde{\sigma}$. Given $k \in \mathbb{R}^{+}$, we define $A_{k}$ as follows. Let $q_{0}, q_{1} \in \tilde{M}$. Define $A_{k}: C_{\widetilde{M}}^{\text {ac }}\left(q_{0}, q_{1}\right) \rightarrow \mathbb{R}$ by

$$
A_{k}(y):=\int_{0}^{T} \frac{1}{2}|\dot{y}(t)|^{2}+k T-\int_{y} \theta .
$$

We define Mañé's action potential $m_{k}: \widetilde{M} \times \widetilde{M} \rightarrow \mathbb{R} \cup\{-\infty\}$ by

$$
m_{k}\left(q_{0}, q_{1}\right):=\inf _{T>0} \inf _{y \in C_{\bar{M}}^{\operatorname{ac}}\left(q_{0}, q_{1} ; T\right)} A_{k}(y) .
$$

Then we have the following result; for a proof see [Contreras and Iturriaga 1999, Proposition 2-1.1] for the first five statements, and [Burns and Paternain 2002, Appendix A] for a proof of the last statement. 
Lemma 2.1. Properties of $m_{k}$ :

(1) If $k \leq k^{\prime}$, then $m_{k}\left(q_{0}, q_{1}\right) \leq m_{k^{\prime}}\left(q_{0}, q_{1}\right)$ for all $q_{0}, q_{1} \in \tilde{M}$.

(2) For all $k \in \mathbb{R}$ and all $q_{0}, q_{1}, q_{2} \in \tilde{M}$, we have

$$
m_{k}\left(q_{0}, q_{2}\right) \leq m_{k}\left(q_{0}, q_{1}\right)+m_{k}\left(q_{1}, q_{2}\right) .
$$

(3) Fix $k \in \mathbb{R}$. Then either $m_{k}\left(q_{0}, q_{1}\right)=-\infty$ for all $q_{0}, q_{1} \in \tilde{M}$, or $m_{k}\left(q_{0}, q_{1}\right) \in \mathbb{R}$ for all $q_{0}, q_{1} \in \widetilde{M}$ and $m_{k}(q, q)=0$ for all $q \in \widetilde{M}$.

(4) If

$$
c(g, \sigma):=\inf \left\{k \in \mathbb{R}: m_{k}\left(q_{0}, q_{1}\right) \in \mathbb{R} \text { for all } q_{0}, q_{1} \in \widetilde{M}\right\},
$$

then $m_{c(g, \sigma)}$ is finite everywhere.

(5) We can alternatively define $c(g, \sigma)$ by

$$
c(g, \sigma)=\inf _{u \in C^{\infty}(\widetilde{M})} \sup _{q \in \widetilde{M}} \frac{1}{2}\left|d_{q} u+\theta_{q}\right|^{2} .
$$

We call the number $c(g, \sigma)$ the Mañe critical value. Using (2-2) it is clear that $c(g, \sigma)<\infty$ if and only if $\theta$ is bounded, that is, if

$$
\|\theta\|_{\infty}:=\sup _{q \in \widetilde{M}}\left|\theta_{q}\right|<\infty .
$$

The functional $S_{\boldsymbol{k}}$. We will now define a second functional $S_{k}$, which will be our main object of study. In the case $c(g, \sigma)<\infty$, it is defined on $\Lambda_{M} \times \mathbb{R}^{+}$. For $c(g, \sigma)=\infty$, it is only defined on $\Lambda_{0} \times \mathbb{R}^{+}$. The following lemma is the key observation required to define $S_{k}$. In the statement, $\mathbb{T}^{2}$ denotes the 2-torus.

Lemma 2.2. If $c(g, \sigma)<\infty$, then $f^{*} \sigma$ is exact for any smooth map $f: \mathbb{T}^{2} \rightarrow M$. Proof. Consider $G:=f_{*}\left(\pi_{1}\left(\mathbb{T}^{2}\right)\right) \leq \pi_{1}(M)$. Then $G$ is amenable since $\pi_{1}\left(\mathbb{T}^{2}\right)=\mathbb{Z}^{2}$, which is amenable. Then [Paternain 2006, Lemma 5.3] says that since $\|\theta\|_{\infty}<\infty$, we can replace $\theta$ by a $G$-invariant primitive $\theta^{\prime}$ of $\tilde{\sigma}$, which descends to define a primitive $\theta^{\prime \prime} \in \Omega^{1}\left(\mathbb{T}^{2}\right)$ of $f^{*} \sigma$.

For each free homotopy class $v \in[\mathbb{T}, M]$, pick a reference loop $x_{v} \in \Lambda_{v}$. Given any $x \in \Lambda_{v}$, let $C(x)$ denote a cylinder with boundary $x(\mathbb{T}) \cup x_{v}(\mathbb{T})$.

Define $S_{k}: \Lambda_{v} \times \mathbb{R}^{+} \rightarrow \mathbb{R}$ by

$$
S_{k}(x, T):=\int_{0}^{1} \frac{1}{2 T}|\dot{x}(t)|^{2} d t+k T-\int_{C(x)} \sigma,
$$

This is well defined because $\int_{C(x)} \sigma$ is independent of the choice of cylinder: If $C^{\prime}(x)$ is another cylinder with the same boundary, then $\mathbb{T}^{2}(x):=C(x) \cup \overline{C^{\prime}(x)}$ is a torus (where $\overline{C^{\prime}(x)}$ denotes the cylinder $C^{\prime}(x)$ taken with the opposite orientation), and $\int_{\mathbb{\mathbb { 2 }}^{2}(x)} \sigma=0$ since $\left.\sigma\right|_{\mathbb{T}^{2}(x)}$ is exact by the previous lemma. 
If $c(g, \sigma)=\infty$, we cannot define $S_{k}$ on all of $\Lambda_{M} \times \mathbb{R}^{+}$, since in this case Lemma 2.2 fails. It is however well defined on $\Lambda_{0} \times \mathbb{R}^{+}$. To see why, consider the case of contractible loops when $c(g, \sigma)<\infty$ again. If $x: \mathbb{T} \rightarrow M$ is contractible and $\boldsymbol{x}: D^{2} \rightarrow M$ denotes a capping disc, so that $\left.\boldsymbol{x}\right|_{\partial D^{2}}=x$, it is easy to see that

$$
\int_{C(x)} \sigma=\int_{D^{2}} x^{*} \sigma
$$

note that the right side is (as it should be) independent of the choice of capping disc $\boldsymbol{x}$, and depends only on $x$ and $\sigma$, since $\left.\sigma\right|_{\pi_{2}(M)}=0$. Moreover the right side is well defined and depends only on $x$ and $\sigma$ even when $c(g, \sigma)=\infty$. Thus it makes sense to define $\left.S_{k}\right|_{\Lambda_{0} \times \mathbb{R}^{+}}$by

$$
S_{k}(x, T)=\int_{0}^{1} \frac{1}{2 T}|\dot{x}(t)|^{2} d t+k T-\int_{D^{2}} \boldsymbol{x}^{*} \sigma ;
$$

this is consistent with the previous definition of $\left.S_{k}\right|_{\Lambda_{0} \times \mathbb{R}^{+}}$when $c(g, \sigma)<\infty$.

Next we will explicitly calculate the derivative of $S_{k}$. Let $\left(x_{s}, T_{s}\right)$ be a variation of $(x, T)$, with $\xi(t):=\left.\frac{\partial}{\partial s}\right|_{s=0} x_{s}(t)$ and $\psi:=\left.\frac{\partial}{\partial s}\right|_{s=0} T_{s}$. Write $E_{q}$ and $E_{v}$ for $\frac{\partial E}{\partial q}$ and $\frac{\partial E}{\partial v}$ respectively. Then an easy calculation in local coordinates shows that the first variation (that is, the Gateaux derivative) of $S_{k}$ at $(\xi, \psi)$, that is, $\left.\frac{\partial}{\partial s}\right|_{s=0} S_{k}\left(x_{s}, T_{s}\right)$ is given by

$$
\begin{aligned}
& \left.\frac{\partial}{\partial s}\right|_{s=0} S_{k}\left(x_{s}, T_{s}\right) \\
& =\psi \int_{0}^{1}(k-E(x(t), \dot{x}(t) / T)) d t+\int_{0}^{1} \sigma_{x(t)}(\xi(t), \dot{x}(t)) d t \\
& \quad \quad+\int_{0}^{1}\left(T \cdot E_{q}(x(t), \dot{x}(t) / T) \cdot \xi(t)+E_{v}(x(t), \dot{x}(t) / T) \cdot \dot{\xi}(t)\right) d t .
\end{aligned}
$$

We claim now that $S_{k}$ is differentiable with respect to the canonical Hilbert manifold structure of $\Lambda_{v} \times \mathbb{R}^{+}$(that is, $S_{k}$ is Fréchet differentiable). In fact, $S_{k}$ is of class $C^{2}$. For this we quote the fact that

$$
(x, T) \mapsto \int_{0}^{1} \frac{1}{2 T}|\dot{x}(t)|^{2} d t+k T
$$

is of class $C^{2}$ (see for instance [Abbondandolo and Schwarz 2009b]) and thus is remains to check that $x \mapsto \int_{C(x)} \sigma$ is differentiable. This can be checked directly. It thus follows that the first variation $\left.\frac{\partial}{\partial s}\right|_{s=0} S_{k}\left(x_{s}, T_{s}\right)$ is actually equal to the (Fréchet) derivative $d_{(x, T)} S_{k}(\xi, \psi)$.

Finally, let us note that

$$
\frac{\partial}{\partial T} S_{k}(x, T)=\frac{1}{T} \int_{0}^{T}(k-E(y, \dot{y})) d t, \quad \text { where } y(t):=x(t / T) .
$$


Relating $S_{\boldsymbol{k}}$ and $\boldsymbol{A}_{\boldsymbol{k}}$. Next, if $(x, T)$ is a critical point of $S_{k}$, then $y(t):=x(t / T)$ satisfies

$$
\int_{0}^{T}\left(E_{q}(y, \dot{y})-\frac{d}{d t} E_{v}(y, \dot{y})\right) \zeta d t-\frac{1}{T} \int_{0}^{T} \sigma_{y}(\zeta, \dot{y}) d t=0,
$$

where $\zeta(t)=\xi(t / T)$. Since this holds for all variations $\zeta$, this implies that if $\tilde{y}:[0, T] \rightarrow \widetilde{M}$ is a lift of $y$, then $\tilde{y}$ satisfies the Euler-Lagrange equations for $L$, that is,

$$
L_{q}(\tilde{y}, \dot{\tilde{y}})-\frac{d}{d t} L_{v}(\tilde{y}, \dot{\tilde{y}})=0 .
$$

Thus $\tilde{y}$ is the lift to $\widetilde{M}$ of the projection to $M$ of an orbit of $\phi_{t}$, and we have the following result.

Corollary 2.3. Let $x \in \Lambda_{M}$ and $\tilde{x}$ denote a lift of $x$ to $\tilde{M}$. Let $T \in \mathbb{R}^{+}$. Define $\tilde{y}(t):=\tilde{x}(t / T)$. Then the following are equivalent:

(1) The pair $(x, T)$ is a critical point of $S_{k}$.

(2) $\tilde{y}$ is a critical point of $A_{k}$.

Thus the pair $(x, T) \in \Lambda_{M} \times \mathbb{R}^{+}$is a critical point of $S_{k}$ if and only if $t \mapsto x(t / T)$ is the projection to $M$ of a closed orbit of $\phi_{t}$.

To specify the lifts we work with, let us fix a lift $\tilde{x}_{v}: I \rightarrow \widetilde{M}$ of $x_{v}$ for each $v \in[\mathbb{T}, M]$. Throughout the paper, given any two paths $y$ and $y^{\prime}$ such that the end point of $y$ is the start point of $y^{\prime}$, the path $y * y^{\prime}$ is the path obtained by first going along $y$ and then going along $y^{\prime}$. Similarly the path $y^{-1}$ is the path obtained by going along $y$ backwards.

Suppose now that $c(g, \sigma)<\infty$. Fix a free homotopy class $v \in[\mathbb{T}, M]$ (which could be the trivial free homotopy class). Let $x \in \Lambda_{v}$, and let $x_{s}$ denote a free homotopy from $x_{0}=x$ to $x_{1}=x_{v}$. Let $z(s):=x_{s}(0)$. Let $\tilde{x}_{s}$ denote the unique homotopy of curves on $\tilde{M}$ that projects down onto $x_{s}$ and satisfies $\tilde{x}_{1}(t)=\tilde{x}_{v}(t)$. Let $\tilde{x}(t):=\tilde{x}_{0}(t), \tilde{z}_{0}(s):=\tilde{x}_{s}(0)$ and $\tilde{z}_{1}(s):=\tilde{x}_{s}(1)$.

Now observe that if $R \subseteq \widetilde{M}$ denotes the rectangle $R=\operatorname{im} \tilde{x}_{s}$, then we have

$$
\int_{C(x)} \sigma=\int_{R} \tilde{\sigma}=\int_{R} d \theta=\int_{\partial R} \theta=\int_{\tilde{x} * \tilde{z}_{1} * \tilde{x}_{v}^{-1} * \tilde{z}_{0}^{-1}} \theta .
$$

Let $\varphi \in \pi_{1}(M)$ denote the unique covering transformation taking $\tilde{z}_{0}$ to $\tilde{z}_{1}$. Since $\langle\varphi\rangle \leq \pi_{1}(M)$ is an amenable subgroup, [Paternain 2006, Lemma 5.3] allows us to assume without loss of generality that $\theta$ is $\varphi$-invariant. Thus $\int_{\tilde{z}_{0}^{-1}} \theta+\int_{\tilde{z}_{1}} \theta=0$. It thus follows that $\int_{C(x)} \sigma=\int_{\tilde{x}} \theta+\int_{\tilde{x}_{v}^{-1}} \theta$.

Set $a_{v}:=\int_{\tilde{x}_{v}^{-1}} \theta$. We conclude that $\int_{C(x)} \sigma=\int_{\tilde{x}} \theta+a_{v}$. This computation shows if $c(g, \sigma)<\infty$, then for any $(x, T) \in \Lambda_{v} \times \mathbb{R}^{+}$, if $\tilde{x}$ is any lift of $x$ and 
$\tilde{y}(t):=\tilde{x}(t / T)$, then

$$
S_{k}(x, T)=A_{k}(\tilde{y})+a_{v} .
$$

For the case $v=0 \in[\mathbb{T}, M]$ the trivial free homotopy class, we may choose the curve $x_{0}$ above to be a constant map, from which it is easy to see that $a_{0}=0$. In particular, if $(x, T) \in \Lambda_{0} \times \mathbb{R}^{+}$and $\tilde{y}$ is defined as before, then

$$
S_{k}(x, T)=A_{k}(\tilde{y}) .
$$

Finally, if $c(g, \sigma)=\infty, S_{k}$ is only defined on $\Lambda_{0} \times \mathbb{R}^{+}$, and it is clear that (2-8) still holds.

\section{The Palais-Smale condition}

Let $(\mathcal{M},\langle\cdot, \cdot\rangle)$ be a Riemannian Hilbert manifold, and suppose $S: \mathcal{M} \rightarrow \mathbb{R}$ is $C^{1}$.

Definition 3.1. We say $S$ satisfies the Palais-Smale condition if every sequence $\left(x_{n}\right) \subseteq \mathcal{M}$ such that $\left\|d_{x_{n}} S\right\| \rightarrow 0$ as $n \rightarrow \infty$ and $\sup _{n}\left|S\left(x_{n}\right)\right|<\infty$ admits a convergent subsequence. We say $S$ satisfies the Palais-Smale condition at the level $\mu \in \mathbb{R}$ if every sequence $\left(x_{n}\right) \subseteq \mathcal{M}$ with $\left\|d_{x_{n}} S\right\| \rightarrow 0$ as $n \rightarrow \infty$ and $S\left(x_{n}\right) \rightarrow \mu$ admits a convergent subsequence.

The following result, concerning $S_{k}$ satisfying the Palais-Smale condition, is adapted from [Contreras 2006, Propositions 3.8 and 3.12]. We will first consider only the case where $c(g, \sigma)<\infty$; see Proposition 3.7 for the case $c(g, \sigma)=\infty$. In the statement of the theorem, $\|\cdot\|$ denotes the operator norm with respect to the metric $\langle\cdot, \cdot\rangle$.

Theorem 3.2. Suppose $c(g, \sigma)<\infty$. Let $A, B, k \in \mathbb{R}^{+}$, and suppose $\left(x_{n}, T_{n}\right) \subseteq$ $\Lambda_{M} \times \mathbb{R}^{+}$satisfies

$$
\sup _{n}\left|S_{k}\left(x_{n}, T_{n}\right)\right| \leq A, \quad \sup _{n} T_{n} \leq B, \quad\left\|d_{\left(x_{n}, T_{n}\right)} S_{k}\right\|<1 / n .
$$

(1) If $\liminf T_{n}>0$, then, passing to a subsequence if necessary, the sequence $\left(x_{n}, T_{n}\right)$ is convergent in the $W^{1,2}$-topology.

(2) If $\lim \inf T_{n}=0$ and the $x_{n}$ are all contractible, then passing to a subsequence if necessary, $S_{k}\left(x_{n}, T_{n}\right) \rightarrow 0$.

Before proving the theorem, let us now fix some notation that we will use throughout this section, as well as implicitly in the rest of the paper. Given a sequence $\left(x_{n}, T_{n}\right) \subseteq \Lambda_{M} \times \mathbb{R}^{+}$, let $y_{n}:\left[0, T_{n}\right] \rightarrow M$ be defined by $y_{n}(t):=x_{n}\left(t / T_{n}\right)$. Define

$$
l_{n}:=\int_{0}^{1}\left|\dot{x}_{n}(t)\right| d t \quad \text { and } \quad e_{n}:=\int_{0}^{1} \frac{1}{2 T_{n}}\left|\dot{x}_{n}(t)\right|^{2} d t
$$


Note that $l_{n}$ is the length of $y_{n}$ and $e_{n}$ is the energy of $y_{n}$. The Cauchy-Schwarz inequality implies

$$
l_{n}^{2} \leq 2 T_{n} e_{n}
$$

Suppose now $c(g, \sigma)<\infty$. Since $\|\theta\|_{\infty}<\infty$, there exist constants $b_{1}, b_{2} \in \mathbb{R}^{+}$ such that

$$
L(q, v) \geq b_{1}|v|^{2}-b_{2} \quad \text { for all }(q, v) \in T \tilde{M} .
$$

Given $A, B, k \in \mathbb{R}^{+}$and a free homotopy class $v \in[\mathbb{T}, M]$, we denote by $\mathbb{D}(A, B, k, v) \subseteq \Lambda_{M} \times \mathbb{R}^{+}$set of pairs $(x, T)$ such that $x \in \Lambda_{v}, \quad S_{k}(x, T) \leq A$ and $T \leq B$.

Proof of Theorem 3.2. We begin with three preparatory lemmas.

Lemma 3.3. Suppose $c(g, \sigma)<\infty$. Let $\left(x_{n}, T_{n}\right) \subseteq \mathbb{D}(A, B, k, v)$. Then if

$$
b(A, B, v):=\frac{A+b_{2} B+\left|a_{v}\right|}{2 b_{1}}
$$

then $e_{n} \leq b(A, B, v)$ for all $n \in \mathbb{N}$.

Proof. We have by (2-7) and (3-2) that

$$
A \geq S_{k}\left(x_{n}, T_{n}\right)=A_{k}\left(\tilde{y}_{n}\right)-a_{v} \geq 2 b_{1} e_{n}-b_{2} T_{n}+k T_{n}+a_{v},
$$

and thus

$$
e_{n} \leq \frac{A+b_{2} T_{n}-k T_{n}+\left|a_{v}\right|}{2 b_{1}} \leq \frac{A+b_{2} B+\left|a_{v}\right|}{2 b_{1}} .
$$

Lemma 3.4. Suppose $c(g, \sigma)<\infty$, and suppose $\left(x_{n}\right) \subseteq \Lambda_{0}$ are such that $l_{n} \rightarrow 0$. Then $\int_{C\left(x_{n}\right)} \sigma \rightarrow 0$.

Proof. Let $\boldsymbol{x}_{n}: D^{2} \rightarrow M$ denote a capping disc for $x_{n}$, so (as in (2-4))

$$
\left.\boldsymbol{x}_{n}\right|_{\partial D^{2}}=x_{n} \text { and } \int_{C\left(x_{n}\right)} \sigma=\int_{D^{2}} \boldsymbol{x}_{n}^{*} \sigma .
$$

Let $\tilde{\boldsymbol{x}}_{n}: D^{2} \rightarrow \widetilde{M}$ denote a lift of $\boldsymbol{x}_{n}$ to $\widetilde{M}$. Then

$$
\left|\int_{D^{2}} \boldsymbol{x}_{n}^{*} \sigma\right|=\left|\int_{D^{2}} \tilde{\boldsymbol{x}}_{n}^{*}(d \theta)\right|=\left|\int_{\tilde{x}_{n}} \theta\right| \leq\|\theta\|_{\infty} l_{n} \rightarrow 0 .
$$

We now reduce Theorem 3.2(1) to a simpler situation:

Lemma 3.5. Suppose $c(g, \sigma)<\infty$ and $\left(x_{n}, T_{n}\right) \in \mathbb{D}(A, B, k, v)$ with $\lim \inf T_{n}>0$. Passing to a subsequence we may assume that there exists $x \in \Lambda_{v}$ such that the $x_{n}$ converge to $x$ in the $C^{0}$-topology. 
Proof. First by compactness of $M$, passing to a subsequence if necessary we may assume there exists $q \in M$ and $T \in \mathbb{R}^{+}$such that $\lim _{n \rightarrow \infty} x_{n}(0)=x_{n}(1)=q$ and $\lim _{n \rightarrow \infty} T_{n}=T$. Consider $g$-geodesics $c_{n}: I \rightarrow M$ such that $c_{n}(0)=q$ and $c_{n}(1)=x_{n}(0)$. By passing to a subsequence we may assume that dist ${ }_{g}\left(x_{n}(0), q\right)<1$, and thus we have $\left|\dot{c}_{n}\right| \leq 1$. Now consider the curves

$w_{n}:\left[0, T_{n}+2\right] \rightarrow M, \quad t \mapsto c_{n} * y_{n} * c_{n}^{-1} \quad$ and $\quad z_{n}: \mathbb{T} \rightarrow M, \quad t \mapsto w_{n}\left(t / T_{n}+2\right)$.

Thus $z_{n}(0)=z_{n}(1)=q$, and $\left(z_{n}\right) \subseteq \Lambda_{v}$.

Given $0 \leq t_{1}<t_{2}<T_{n}+2$,

$\operatorname{dist}_{g}\left(w_{n}\left(t_{1}\right), w_{n}\left(t_{2}\right)\right) \leq \int_{t_{1}}^{t_{2}}\left|\dot{w}_{n}(t)\right| d t \leq \sqrt{2}\left|t_{2}-t_{1}\right|^{1 / 2}\left(\int_{0}^{T_{n}+2} \frac{1}{2}\left|\dot{w}_{n}(t)\right|^{2} d t\right)^{1 / 2}$.

By Lemma 3.3 we have

$$
\int_{0}^{T_{n}+2} \frac{1}{2}\left|\dot{w}_{n}(t)\right|^{2} d t=\int_{0}^{1} \frac{1}{2}\left|\dot{c}_{n}(t)\right|^{2} d t+e_{n}+\int_{0}^{1} \frac{1}{2}\left|\dot{c}_{n}^{-1}(t)\right|^{2} d t \leq 1+b(A, B, v),
$$

and thus $\operatorname{dist}_{g}\left(w_{n}\left(t_{1}\right), w_{n}\left(t_{2}\right)\right) \leq \sqrt{2}\left|t_{2}-t_{1}\right|^{1 / 2}(1+b(A, B, v))^{1 / 2}$. Hence the family $\left(w_{n}\right)$ is equicontinuous. The Arzelà-Ascoli theorem then completes the proof.

Proof of Theorem 3.2. We begin by proving Theorem 3.2(1). This part of the proof is very similar to the proof of [Contreras et al. 2000, Theorem B]. Suppose $\left(x_{n}, T_{n}\right) \subseteq \mathbb{D}(A, B, k, v)$ with $\lim \inf T_{n}>0$. By the previous lemma, after passing to a subsequence if necessary, we may assume that $\left(x_{n}, T_{n}\right)$ converges in the $C^{0}$ topology to some $(x, T)$, where $T>0$.

Without loss of generality, let us assume that the limit curve $x$ is contained in a single chart $U$ (otherwise simply repeat these arguments finitely many times). Then after passing possibly to another subsequence, we may assume that the $x_{n}$ are all contained in $U$ as well. There exists a constant $b_{3} \in \mathbb{R}^{+}$such that in the coordinates on $U$,

$$
b_{3}:=\sup _{q \in U, v \in T_{q} M} \frac{\left|E_{q}(q, v)\right|}{1+|v|^{2}}<\infty .
$$

Write $z_{n}(t):=T_{n}^{-1} x_{n}(t)$. By Lemma 3.3 we can find a constant $R>0$ such that

$$
\left|x_{n}\right|_{1,2} \leq R \quad \text { and } \quad\left|z_{n}\right|_{1,2} \leq R .
$$

Now since $\left\|d_{\left(x_{n}, T_{n}\right)} S_{k}\right\| \rightarrow 0$ as $n \rightarrow \infty$, given $\varepsilon>0$ there exists $N \in \mathbb{N}$ such that for every $(\xi, \psi)$ satisfying $|(\xi, \psi)| \leq 2 R$ and $n, m \geq N$, we have

$$
\left|d_{\left(x_{n}, T_{n}\right)} S_{k}(\xi, \psi)-d_{\left(x_{m}, T_{m}\right)} S_{k}(\xi, \psi)\right|<\varepsilon .
$$


Take $\xi=x_{n}-x_{m}$ and $\psi=0$ and use (2-5) to discover that

$$
\begin{aligned}
\mid \int_{0}^{1}\left(T_{n} \cdot E_{q}\left(x_{n}, \dot{z}_{n}\right)-T_{m} \cdot E_{q}\left(x_{m}, \dot{z}_{m}\right)\right)\left(x_{n}-x_{m}\right) d t & \\
+\int_{0}^{1}\left(E_{v}\left(x_{n}, \dot{z}_{n}\right)-\right. & \left.E_{v}\left(x_{m}, \dot{z}_{m}\right)\right)\left(\dot{x}_{n}-\dot{x}_{m}\right) d t \\
& +\int_{0}^{1} \sigma_{x_{n}}\left(\dot{x}_{n}, \dot{x}_{m}\right)-\sigma_{x_{m}}\left(\dot{x}_{n}, \dot{x}_{m}\right) d t \mid<\varepsilon .
\end{aligned}
$$

Here we are using the canonical parallel transport available to us on Euclidean spaces to view $\dot{x}_{n}-\dot{x}_{m}$ as a tangent vector in any tangent space of our choosing. Using (3-3) we can bound the first integral as follows:

$$
\begin{aligned}
\mid \int_{0}^{1}\left(T_{n} \cdot E_{q}\left(x_{n}, \dot{z}_{n}\right)-T_{m} \cdot E_{q}\left(x_{m}, \dot{z}_{m}\right)\right) & \left(x_{n}-x_{m}\right) d t \mid \\
& \leq\left(2 B b_{3}+2 b_{3} b(A, B, v)\right)\left\|x_{n}-x_{m}\right\|_{\infty} .
\end{aligned}
$$

Let us write $\left.\sigma\right|_{U}$ in local coordinates as $\sigma=\sigma_{i j} d q^{i} \wedge d q^{j}$, where $\sigma_{i j} \in C^{\infty}(U, \mathbb{R})$. Then since

$$
\left|\sigma_{i j}\left(x_{n}(t)\right)-\sigma_{i j}\left(x_{m}(t)\right)\right| \rightarrow 0 \quad \text { as } n, m \rightarrow \infty \text {, uniformly in } t,
$$

and

$$
\int_{0}^{1}\left|\dot{x}_{n}\right|\left|\dot{x}_{m}\right| d t \leq 2 \sqrt{T_{n} T_{m} e_{n} e_{m}}
$$

is bounded, it follows that for $n$ and $m$ large the third integral is small. Thus the second integral must also be small for large $n$ and $m$. Since

$$
\left|v-v^{\prime}\right|^{2}=\left(E_{v}(q, v)-E_{v}\left(q^{\prime}, v^{\prime}\right)\right) \cdot\left(v-v^{\prime}\right),
$$

we have

$$
\int_{0}^{1}\left|\dot{z}_{n}-\dot{z}_{m}\right|^{2} d t \leq \int_{0}^{1}\left(E_{v}\left(x_{n}, \dot{z}_{n}\right)-E_{v}\left(x_{m}, \dot{z}_{m}\right)\right)\left(\dot{z}_{n}-\dot{z}_{m}\right) d t,
$$

and hence the fact that the second integral in (3-4) is small for large $n$ and $m$ implies that the sequence $\left(z_{n}\right)$, and hence the sequence $\left(x_{n}\right)$, converges in the $W^{1,2}$ topology. This completes the proof of Theorem 3.2(1).

We now prove Theorem 3.2(2). This part of the proof follows the proof of [Contreras 2006, Theorem 3.8] very closely. Assume $\left(x_{n}, T_{n}\right) \subseteq \mathbb{D}(A, B, k, 0)$ (where $0 \in[\mathbb{T}, M]$ denotes the trivial free homotopy class) and that $\lim \inf T_{n}=0$. Passing to a subsequence, we may assume that $T_{n} \rightarrow 0$. It suffices to show that passing to a subsequence we have $e_{n} \rightarrow 0$. Then by Lemma 3.4, $S_{k}\left(x_{n}, T_{n}\right)=$ $e_{n}+k T_{n}-\int_{C\left(x_{n}\right)} \sigma \rightarrow 0$. 
We know that $e_{n}$ remains bounded by Lemma 3.3. Since $T_{n} \rightarrow 0$, (3-1) implies that $l_{n} \rightarrow 0$. Thus as before we may assume that all the curves $x_{n}$ take their image in the domain of some chart $U$ on $M$. Thus for the remainder of the proof we work in coordinates as if $M=\mathbb{R}^{d}$. Let $\xi_{n}(t):=x_{n}(t)-x_{n}(0)$, so that $\xi_{n}(0)=\xi_{n}(1)=0$. Then $\left(\xi_{n}, 0\right) \in T_{\left(x_{n}, T_{n}\right)}\left(\Lambda_{\mathbb{R}^{d}} \times \mathbb{R}^{+}\right)$. Let also $\zeta_{n}(t):=\xi_{n}\left(t / T_{n}\right)$, so that $\dot{\zeta}_{n}(t)=\dot{y}_{n}(t)$. Then

$$
\left|d_{\left(x_{n}, T_{n}\right)} S_{k}\left(\xi_{n}, 0\right)\right| \leq \frac{1}{n}\left(T_{n} \int_{0}^{T_{n}}\left|\dot{\zeta}_{n}(t)\right|^{2} d t\right)^{1 / 2} \leq \frac{1}{n} \sqrt{2 T_{n} e_{n}}
$$

Using (2-5) we have

$$
\begin{aligned}
d_{\left(x_{n}, T_{n}\right)} S_{k}\left(\xi_{n}, 0\right)=\int_{0}^{T_{n}}\left(E_{q}\left(y_{n}, \dot{y}_{n}\right) \cdot \zeta_{n}+E_{v}\left(y_{n}, \dot{y}_{n}\right) \cdot \dot{\zeta}_{n}(t)\right) d t & \\
& +\int_{0}^{1} \sigma_{x_{n}(t)}\left(\xi_{n}(t), \dot{x}_{n}(t)\right) d t .
\end{aligned}
$$

There exists $b_{4} \in \mathbb{R}^{+}$such that

$$
\left|\int_{0}^{1} \sigma_{x_{n}(t)}\left(\xi_{n}(t), \dot{x}_{n}(t)\right) d t\right| \leq b_{4} \int_{0}^{1}\left|\xi_{n}(t)\right|\left|\dot{x}_{n}(t)\right| d t \leq b_{4} l_{n}^{2} .
$$

Thus using (3-3) and the fact that $E_{v}(q, v) \cdot \xi=\langle v, \xi\rangle$, we have

$$
\begin{aligned}
d_{\left(x_{n}, T_{n}\right)} S_{k}\left(\xi_{n}, 0\right) & \geq-b_{3} \int_{0}^{T_{n}}\left(1+\left|\dot{y}_{n}(t)\right|^{2}\right)\left|y_{n}(t)-y_{n}(0)\right| d t+2 e_{n}-b_{4} l_{n}^{2} \\
& \geq-b_{3} l_{n}\left(T_{n}+2 e_{n}\right)+2 e_{n}-b_{4} l_{n}^{2} .
\end{aligned}
$$

Putting this together and dividing through by $\sqrt{T_{n}}$, we have

$$
-b_{3} l_{n} \sqrt{T_{n}}-2 b_{3} \frac{e_{n} l_{n}}{\sqrt{T_{n}}}+2 \frac{e_{n}}{\sqrt{T_{n}}}-b_{4} \frac{l_{n}^{2}}{\sqrt{T_{n}}} \leq \frac{1}{n} \sqrt{2 e_{n}} .
$$

By (3-1), we have

$$
\lim _{n \rightarrow \infty} \frac{l_{n}^{2}}{\sqrt{T_{n}}}=0 \quad \text { and } \quad \lim _{n \rightarrow \infty} \frac{l_{n}}{\sqrt{T_{n}}} \text { is bounded; }
$$

thus $\lim _{n \rightarrow \infty} e_{n} / \sqrt{T_{n}}$ must also be bounded, and this can happen if and only if $e_{n} \rightarrow 0$. This completes the proof of Theorem 3.2(2).

We now wish to study the case where $c(g, \sigma)=\infty$. Recall in this case $S_{k}$ is only defined on $\Lambda_{0} \times \mathbb{R}^{+}$. For a result similar to Theorem 3.2 to hold in the unbounded setting, we must restrict to a subset of $\Lambda_{0} \times \mathbb{R}^{+}$.

Definition 3.6. Suppose $K \subseteq \widetilde{M}$ is compact. Define $\Lambda_{0}^{K} \subseteq \Lambda_{0}$ to be the set of loops $x \in \Lambda_{0}$ such that there exists a lift $\tilde{x}: \mathbb{T} \rightarrow \widetilde{M}$ of $x$ such that $\tilde{x}(\mathbb{T}) \subseteq K$.

Here is the extension of Theorem 3.2 to the case $c(g, \sigma)=\infty$. 
Proposition 3.7. Suppose that $c(g, \sigma)=\infty$. Let $A, B, k \in \mathbb{R}^{+}$and take $K \subseteq \tilde{M}$ to be compact. Suppose $\left(x_{n}, T_{n}\right) \subseteq \Lambda_{0}^{K} \times \mathbb{R}^{+}$satisfy

$$
\sup _{n}\left|S_{k}\left(x_{n}, T_{n}\right)\right| \leq A, \quad \sup _{n} T_{n} \leq B, \quad\left\|d_{\left(x_{n}, T_{n}\right)} S_{k}\right\|<1 / n .
$$

(1) If $\liminf T_{n}>0$, then passing to a subsequence if necessary the sequence $\left(x_{n}, T_{n}\right)$ is convergent in the $W^{1,2}$-topology.

(2) If $\liminf T_{n}=0$, then passing to a subsequence if necessary it holds that $S_{k}\left(x_{n}, T_{n}\right) \rightarrow 0$.

Proof. The proof proceeds exactly as before, since any primitive $\theta$ of $\tilde{\sigma}$ is bounded on $K$.

\section{Supercritical energy levels: The case $k>c(g, \sigma)$}

We now assume $c(g, \sigma)<\infty$, and study supercritical energies $k>c(g, \sigma)$. We aim to prove Theorem 1.1(1). The key fact we will use is the following result. As before, let $(\mathcal{M},\langle\cdot, \cdot\rangle)$ be a Riemannian Hilbert manifold, and let $S: \mathcal{M} \rightarrow \mathbb{R}$ be of class $C^{1}$.

Proposition 4.1. Suppose $S$ is bounded from below and satisfies the Palais-Smale condition, and suppose for every $A \in \mathbb{R}^{+}$the set $\{x \in \mathcal{M}: S(x) \leq A\}$ is complete. Then $S$ has a global minimum.

A proof may be found in [Contreras et al. 2000, Corollary 23]. Fix a nontrivial free homotopy class $v \in[\mathbb{T}, M]$. The aim of this section is to verify for $k>c(g, \sigma)$ that the functional $S_{k}$ on the Hilbert manifold $\Lambda_{v} \times \mathbb{R}^{+}$satisfies the hypotheses of Proposition 4.1, for then the global minimum whose existence Proposition 4.1 guarantees is our desired closed orbit of energy $k$.

The first step then is the following lemma, whose proof only requires $k \geq c(g, \sigma)$, and works for any free homotopy class $v \in[\mathbb{T}, M]$.

Lemma 4.2. Let $k \geq c(g, \sigma)$. Then $\left.S_{k}\right|_{\Lambda_{v} \times \mathbb{R}^{+}}$is bounded below.

Proof. The argument begins by replicating an argument seen earlier in Section 2. Fix a free homotopy class $v \in[\mathbb{T}, M]$ (which could be the trivial free homotopy class). Let $(x, T) \in \Lambda_{v} \times \mathbb{R}^{+}$, and let $x_{s}$ denote a free homotopy from $x_{0}=x$ to $x_{1}=x_{v}$. Let $z(s):=x_{s}(0)$. Lift $x_{s}$ to a homotopy $\tilde{x}_{s}$ in $\tilde{M}$ with $\tilde{x}_{1}(t)=\tilde{x}_{v}(t)$, and let $\tilde{x}(t):=\tilde{x}_{0}(t), \tilde{z}_{0}(s)=\tilde{x}_{s}(0)$ and $\tilde{z}_{1}(s)=\tilde{x}_{s}(1)$.

Now observe that if $R \subseteq \widetilde{M}$ denotes the rectangle $R=\operatorname{im} \tilde{x}_{s}$, then we have

$$
\int_{C(x)} \sigma=\int_{R} \tilde{\sigma}=\int_{R} d \theta=\int_{\partial R} \theta=\int_{\tilde{x} * \tilde{z}_{1} * \tilde{x}_{\nu}^{-1} * \tilde{z}_{0}^{-1}} \theta .
$$

Suppose $\varphi \in \pi_{1}(M)$ denotes the unique covering transformation taking $\tilde{z}_{0}$ to $\tilde{z}_{1}$. Since $\langle\varphi\rangle \leq \pi_{1}(M)$ is an amenable subgroup, [Paternain 2006, Lemma 5.3] allows 
us to assume that without loss of generality, $\theta$ is $\varphi$-invariant. Thus $\int_{\tilde{z}_{0}^{-1}} \theta+\int_{\tilde{z}_{1}} \theta=0$. It thus follows that

$$
\int_{C(x)} \sigma=\int_{\tilde{x}} \theta+\int_{\tilde{x}_{v}^{-1}} \theta
$$

Let $\tilde{x}_{n}:=\varphi^{n} \tilde{x}$, and use similar notations for $\tilde{z}_{n}$ and $\tilde{x}_{v, n}$. Let $\tilde{y}_{n}:=\tilde{x}_{n}(t / T)$, so $\tilde{y}_{n}:[0, T] \rightarrow \widetilde{M}$. Then for any $n \in \mathbb{N}$ we consider the closed loop $u_{n}:\left[0, T_{n}\right] \rightarrow \widetilde{M}$ defined by

$$
u_{n}=\tilde{y}_{0} * \tilde{y}_{1} * \cdots * \tilde{y}_{n} * \tilde{z}_{n+1} * \tilde{x}_{v, n}^{-1} * \cdots * \tilde{x}_{v, 1}^{-1} * \tilde{x}_{v}^{-1} * \tilde{z}_{0}^{-1},
$$

where $T_{n}:=(n+1) T+1+(n+1)+1$. We have

$$
\begin{aligned}
A_{k}\left(u_{n}\right)=(n+1)\left(\int_{0}^{T} \frac{1}{2}|\dot{\tilde{y}}(t)|^{2} d t\right. & \left.+\int_{0}^{1} \frac{1}{2}\left|\dot{\tilde{x}}_{v}^{-1}\right|^{2} d t-\int_{\tilde{y}_{0}} \theta-\int_{\tilde{x}_{v}^{-1}} \theta\right) \\
& +\int_{0}^{1} \frac{1}{2}\left|\dot{\tilde{z}}_{1}(t)\right|^{2} d t+\int_{0}^{1} \frac{1}{2}\left|\dot{\tilde{z}}_{0}^{-1}(t)\right|^{2} d t+k T_{n} .
\end{aligned}
$$

Now if $k \geq c(g, \sigma)$, then by definition of $c(g, \sigma)$ we have $A_{k}\left(u_{n}\right) \geq 0$. Thus,

$$
\begin{aligned}
0 \leq \int_{0}^{T} \frac{1}{2}\left|\dot{\tilde{y}}_{0}(t)\right|^{2} d t+\int_{0}^{1} \frac{1}{2}\left|\dot{\tilde{x}}_{v}^{-1}\right|^{2} d t & \int_{\tilde{y}_{0}} \theta-\int_{\tilde{x}_{v}^{-1}} \theta+\frac{k T_{n}}{n+1} \\
& +\frac{1}{n+1}\left(\int_{0}^{1}\left|\dot{\tilde{z}}_{1}(t)\right|^{2} d t+\int_{0}^{1}\left|\dot{\tilde{z}}_{0}^{-1}(t)\right|^{2} d t\right) .
\end{aligned}
$$

Letting $n \rightarrow \infty$ and substituting for the terms with $\tilde{y}_{0}$ we obtain

$$
\int_{0}^{1} \frac{1}{2 T}|\dot{x}(t)|^{2} d t+\int_{0}^{1} \frac{1}{2}\left|\dot{x}_{v}^{-1}\right|^{2} d t-\int_{\tilde{x}} \theta-a_{v}+k(T+1) \geq 0 .
$$

Now

$$
\begin{aligned}
S_{k}(x, T) & =\int_{0}^{1} \frac{1}{2 T}|\dot{x}(t)|^{2} d t+k T-\int_{C(x)} \sigma \\
& =\int_{0}^{1} \frac{1}{2 T}|\dot{x}(t)|^{2} d t+k T-\int_{\tilde{x}} \theta-a_{\nu},
\end{aligned}
$$

and hence by (4-1) and (4-2),

$$
S_{k}(x, T)+\int_{0}^{1} \frac{1}{2}\left|\dot{\tilde{x}}_{v}^{-1}(t)\right|^{2} d t+k \geq 0,
$$

that is,

$$
S_{k}(x, T) \geq-\int_{0}^{1} \frac{1}{2}\left|\dot{\tilde{x}}_{v}(t)^{-1}\right|^{2} d t-k>-\infty .
$$


Let us set $i_{k, v}:=\inf _{(x, T) \in \Lambda_{v} \times \mathbb{R}^{+}} S_{k}(x, T)$, so that the lemma tells us $i_{k, v}>-\infty$ for $k \geq c(g, \sigma)$.

The next lemma implies that $\left\{\left.S_{k}\right|_{\Lambda_{v} \times \mathbb{R}^{+}} \leq A\right\}$ is complete for any $A \geq 0$.

Lemma 4.3. Suppose $c(g, \sigma)<\infty$. Let $v \in[\mathbb{T}, M]$ be a nontrivial free homotopy class and let $A \in \mathbb{R}^{+}$. There exists $T_{0}=T_{0}(A, k, v) \in \mathbb{R}^{+}$such that $T \geq T_{0}$ if $(x, T) \in \mathbb{D}(A, \infty, k, v)$.

Proof. Let $\tilde{x}$ denote a lift of $x$ and let $\tilde{y}:[0, T] \rightarrow \tilde{M}$ be the curve $t \mapsto \tilde{x}(t / T)$. Using (2-7) and (3-2), we compute that

$A \geq S_{k}(x, T)=A_{k}(\tilde{y})+a_{v} \geq \frac{b_{1}}{T} \int_{0}^{1}|\dot{\tilde{x}}|^{2} d t-\left(k-b_{2}\right) T+a_{v} \geq \frac{b_{1}}{T} l(v)-\left(k-b_{2}\right) T+a_{v}$,

where $l(v):=\inf \left\{\int_{0}^{1}|\dot{x}(t)| d t: x \in \Lambda_{v}\right\}$. Since $M$ is closed and $v$ is a nontrivial free homotopy class, we have $l(v)>0$, which implies the lemma.

Proof of Theorem 1.1(1). Take $k>c(g, \sigma)$, and fix a nontrivial free homotopy class $v \in[\mathbb{T}, M]$. Let $\left(x_{n}, T_{n}\right) \subseteq \mathbb{D}(A, \infty, k, v)$. We want to show that $\left(x_{n}, T_{n}\right)$ admits a convergent subsequence in the $W^{1,2}$-topology. In view of Theorem 3.2, it suffices to show that there exists $B>0$ such that $\left(x_{n}, T_{n}\right) \subseteq \mathbb{D}(A, B, k, v)$ and that $\lim \inf T_{n}>0$.

Lemma 4.4. The sequence $\left(T_{n}\right)$ is bounded above and bounded away from zero.

Proof. First we claim that $\left(T_{n}\right)$ is bounded. Indeed, if $c=c(g, \sigma)$,

$$
A \geq S_{k}\left(x_{n}, T_{n}\right)=S_{c}\left(x_{n}, T_{n}\right)+(k-c) T_{n} \geq i_{c, v}+(k-c) T_{n},
$$

and thus $\left(T_{n}\right)$ is bounded. Say $T_{n} \leq B$ for all $n$, where $B \in \mathbb{R}^{+}$. Passing to a subsequence we may assume that if $T:=\liminf T_{n}$, then $T_{n} \rightarrow T$. It remains to check $T>0$. From (3-1) and Lemma 3.3 if $T=0$, then $l_{n} \rightarrow 0$. This is a contradiction since $l_{n}>l(v)>0$ (see the proof of the previous lemma).

\section{Subcritical energy levels: The case $k<c(g, \sigma)$}

In this section we drop the assumption that $c(g, \sigma)<\infty$, and study subcritical energies $k<c(g, \sigma)$.

Mountain pass geometry. Again let $(\mathcal{M},\langle\cdot, \cdot\rangle)$ be a Riemannian Hilbert manifold and $S: \mathcal{M} \rightarrow \mathbb{R}$ a function of class $C^{2}$. Let $\Phi_{s}$ denote the (local) flow of $-\nabla S$. Define $\alpha: \mathcal{M} \rightarrow \mathbb{R}^{+} \cup\{\infty\}$ by

$$
\alpha(x):=\sup \left\{r>0: s \mapsto \Phi_{s}(x) \text { is defined on }[0, r]\right\} .
$$

An admissible time is a differentiable function $\tau: \mathcal{M} \rightarrow \mathbb{R}$ such that $0 \leq \tau(x)<\alpha(x)$ for all $x \in \mathcal{M}$. 
Let $\mathscr{F}$ denote a family of subsets of $\mathcal{M}$, and define $\mu:=\inf _{F \in \mathscr{F}} \sup _{x \in F} S(x)$. Suppose that $\mu \in \mathbb{R}$. We say that $\mathscr{F}$ is $S$-forward invariant if the following holds: if $\tau$ is an admissible time such that $\tau(x)=0$ if $S(x) \leq \mu-\delta$ for some $\delta>0$, then for all $F \in \mathscr{F}_{F}$ the set $F_{\tau}:=\left\{\Phi_{\tau(x)}(x): x \in F\right\}$ is also a member of $\mathscr{F}_{F}$.

For convenience, given a subset $\mathscr{V} \subseteq \mathcal{M}$ and $a \in \mathbb{R}$, let $K_{a, \mathscr{V}}:=\operatorname{crit} S \cap S^{-1}(a) \cap \mathcal{V}$ denote the set of critical points of $S$ in $\mathscr{V}$ at the level $a$.

Our main tool will be the following mountain pass theorem, whose statement is similar to that of [Contreras 2006, Proposition 6.3]. In what follows, a strict local minimizer of a function $S: \mathcal{M} \rightarrow \mathbb{R}$ is a point $x \in \mathcal{M}$ such that there exists a neighborhood $\mathcal{N}$ of $x$ such that $S(y)>S(x)$ for all $y \in \mathcal{N} \backslash\{x\}$.

Theorem 5.1. Let $\mathcal{M}$ be a Riemannian Hilbert manifold and $S: \mathcal{M} \rightarrow \mathbb{R}$ a function of class $C^{2}$. Suppose we are given a sequence $\left(\mathscr{F}_{n}\right)$ offamilies of subsets of $\mathcal{M}$ with $\mathscr{F}_{n} \subseteq \mathscr{F}_{n+1}$ for all $n \in \mathbb{N}$. Set $\mathscr{F}_{\infty}:=\bigcup_{n} \mathscr{F}_{n}$. Set $\mu_{\infty}:=\inf _{F \in \mathscr{F}_{\infty}} \sup _{x \in F} S(x)$. Suppose in addition that

(1) $\mathscr{F}_{\infty}$ is $S$-forward invariant, and the sets $F \in \mathscr{F}_{\infty}$ are connected;

(2) $\mu_{\infty} \in \mathbb{R}$;

(3) the flow $\Phi_{s}$ of $-\nabla S$ is relatively complete on $\left\{\mu_{\infty}-\eta \leq S \leq \mu_{\infty}+\eta\right\}$ for some $\eta>0$;

(4) there are closed subsets $\left(\cup_{n}\right)$ of $\mathcal{M}$ such that for all $\varepsilon>0$, there exists $n(\varepsilon) \in \mathbb{N}$ such that for all $n \geq n(\varepsilon)$ there exists $F \in \mathscr{F}_{n}$ and $0<\varepsilon_{1}(n)<\varepsilon$ such that

$$
F \subseteq\left\{S \leq \mu_{\infty}-\varepsilon_{1}(n)\right\} \cup\left(\mathcal{U}_{n} \cap\left\{S \leq \mu_{\infty}+\varepsilon\right\}\right) ; \quad \text { and }
$$

(5) there are closed subsets $\left(\mathscr{V}_{n}\right)$ and a sequence $\left(r_{n}\right) \subseteq \mathbb{R}^{+}$such that

$$
\mathscr{P}_{r_{n}}\left(\mathcal{U}_{n}\right):=\left\{x \in \mathcal{M}: \operatorname{dist}\left(x, \mathscr{U}_{n}\right)<r_{n}\right\} \subseteq \mathscr{V}_{n},
$$

and such that $\left.S\right|_{\gamma_{n}}$ satisfies the Palais-Smale condition at the level $\mu_{\infty}$.

Then if $\mathscr{V}_{\infty}:=\bigcup_{n \in \mathbb{N}} \mathscr{V}_{n}$, then $S$ has a critical point $x \in \mathscr{V}_{\infty}$ with $S(x)=\mu_{\infty}$, that is, $K_{\mu_{\infty}, \mathscr{V}_{\infty}} \neq \varnothing$. Moreover, if

$$
\sup _{F \in \mathscr{F}_{\infty}} \inf _{x \in F} S(x)<\mu_{\infty},
$$

then there is a point in $K_{\mu_{\infty}, V_{\infty}}$ that is not a strict local minimizer of $S$.

The proof is an easy application of the following result, which can be found as [Contreras 2006, Lemma 6.2].

Lemma 5.2. Let $\mathcal{M}$ be a Riemannian Hilbert manifold and let $\mathcal{U} \subseteq \mathscr{V} \subseteq \mathcal{M}$ be closed subsets such that $\mathscr{B}_{r}(\mathcal{U}) \subseteq \mathcal{V}$ for some $r>0$. Let $S: M \rightarrow \mathbb{R}$ be a $C^{2}$ function, and let $\mu \in \mathbb{R}$ be such that $\left.S\right|_{\gamma}$ satisfies the Palais-Smale condition at the level $\mu$. 
Suppose also that the flow $\Phi_{s}$ of $-\nabla S$ is relatively complete on $\{|S-\mu| \leq \eta\}$ for some $\eta>0$.

Then if $\mathcal{N}$ is any neighborhood of $K_{\mu, \mathcal{V}}$ relative to $\mathscr{V}$, then for any $\lambda>0$ there exists $\varepsilon$ and $\delta$ with $0<\varepsilon<\delta<\lambda$ such that for any $0<\varepsilon_{1}<\varepsilon$ there exists an admissible time $\tau$ such that $\tau(x)=0$ for all $x \in\{|S-\mu| \geq \delta\}$, and such that if

$$
F:=\left\{S \leq \mu-\varepsilon_{1}\right\} \cup(U \cap\{S \leq \mu+\varepsilon\}),
$$

then $F_{\tau} \subseteq \mathcal{N} \cup\left\{S \leq \mu-\varepsilon_{1}\right\}$.

Proof of Theorem 5.1. We will show that $K_{\mu_{\infty}, \vartheta_{n}} \neq \varnothing$ for $n$ large enough. Fix $0<\varepsilon<\delta<\lambda:=1$ as in the statement of Lemma 5.2. By hypothesis there exists $n(\varepsilon) \in \mathbb{N}$ such that for all $n \geq n(\varepsilon)$ there exists $0<\varepsilon_{1}(n)<\varepsilon$ and $F \in \mathscr{F}_{n}$ such that

$$
F \subseteq\left\{S \leq \mu_{\infty}-\varepsilon_{1}(n)\right\} \cup\left(u_{n} \cap\left\{S \leq \mu_{\infty}+\varepsilon\right\}\right) .
$$

For such $n$, we have $K_{\mu_{\infty}, V_{n}} \neq \varnothing$. Indeed, if $K_{\mu_{\infty}, \mathscr{V}_{n}}=\varnothing$, by Lemma 5.2, there exists an admissible time $\tau$ such that $\tau \equiv 0$ on $\left\{S \leq \mu_{\infty}-\delta\right\}$, and such that $F_{\tau}$ satisfies $F_{\tau} \subseteq\left\{S \leq \mu_{\infty}-\varepsilon_{1}(n)\right\}$ (for we may take $\mathcal{N}=\varnothing$ in Lemma 5.2). Since $\mathscr{F}_{\infty}$ is forward invariant, $F_{\tau} \in \mathscr{F}_{\infty}$. This contradicts the definition of $\mu_{\infty}$.

To prove the last statement, suppose that $K_{\mu_{\infty}, V_{\infty}}$ consists entirely of strict local minimizers, and that (5-1) holds. Choose $\lambda_{0}>0$ such that

$$
\sup _{F \in \mathscr{F}_{\infty}} \inf _{x \in F} S(x)<\mu_{\infty}-2 \lambda_{0} .
$$

For each $x \in K_{\mu_{\infty}, V_{\infty}}$, let $\mathcal{N}(x)$ denote a neighborhood of $x$ such that $S(y)>S(x)$ for all $y \in \mathcal{N}(x) \backslash\{x\}$, and let

$$
\mathcal{N}_{0}:=\bigcup_{x \in K_{\mu_{\infty}, V_{\infty}}} \mathcal{N}(x) \text { and } \quad \mathcal{N}_{n}:=\mathcal{N}_{0} \cap \mathcal{V}_{n} \text { for each } n \in \mathbb{N} .
$$

Let $0<\varepsilon<\delta<\lambda_{0}$ be given by Lemma 5.2 for $\mathcal{N}_{0}$. By hypothesis there exists $n(\varepsilon) \in \mathbb{N}$ such that for all $n \geq n(\varepsilon)$ there exists $0<\varepsilon_{1}(n)<\varepsilon$ and $F \in \mathscr{F}_{n}$ such that

$$
F \subseteq\left\{S \leq \mu_{\infty}-\varepsilon_{1}(n)\right\} \cup\left(u_{n} \cap\left\{S \leq \mu_{\infty}+\varepsilon\right\}\right) .
$$

By Lemma 5.2, there exists an admissible time $\tau$ such that $\tau \equiv 0$ on $\left\{S \leq \mu_{\infty}-\delta\right\}$ and such that $F_{\tau} \subseteq \mathcal{N}_{n} \cup\left\{S \leq \mu_{\infty}-\varepsilon_{1}(n)\right\} \subseteq \mathcal{N}_{0} \cup\left\{S \leq \mu_{\infty}-\varepsilon_{1}(n)\right\}$. By definition of $\mathcal{N}_{0}$, the sets $\mathcal{N}_{0}$ and $\left\{S \leq \mu_{\infty}-\varepsilon_{1}(n)\right\}$ are disjoint, so $\mathcal{N}_{0} \cup\left\{S \leq \mu_{\infty}-\varepsilon_{1}(n)\right\}$ is disconnected. Since $F_{\tau}$ is connected by hypothesis, we either have $F_{\tau} \subseteq \mathcal{N}_{0}$ and $F_{\tau} \cap\left\{S \leq \mu_{\infty}-\varepsilon_{1}(n)\right\}=\varnothing$, or $F_{\tau} \subseteq\left\{S \leq \mu_{\infty}-\varepsilon_{1}(n)\right\}$. The former fails since $\varepsilon_{1}(n)<\varepsilon<\lambda_{0}$, and the value of $S$ decreases under $\Phi_{s}$, and the latter contradicts the definition of $\mu_{\infty}$. 
Proof of the second statement of Theorem 1.1(2). The main tool we will use will be Theorem 5.1. The first step however is the following result, whose statement and proof closely parallel [Contreras 2006, Proposition C].

Proposition 5.3. Let $k \in \mathbb{R}^{+}$. Then there exists a constant $\mu_{0}>0$ such that if $f: I \rightarrow \Lambda_{0} \times \mathbb{R}^{+}$is any path such that, with $f(0)=\left(x_{0}, T_{0}\right)$ and $f(1)=\left(x_{1}, T_{1}\right)$, we have

(1) $S_{k}\left(x_{0}, T_{0}\right)<0$, and

(2) $x_{1}$ is the constant curve $x_{1}(t) \equiv x_{0}(0)$,

then $\sup _{s \in I} S_{k}(f(s))>\mu_{0}>0$.

Remark. The constant $\mu_{0}$ does not depend on $T_{1}$.

In the statement of the following, as before, we put $l(x):=\int_{0}^{1}|\dot{x}(t)| d t$.

Lemma 5.4 [Contreras 2006, Lemma 5.1]. Let $\theta \in \Omega^{1}(\widetilde{M})$. Given any $q \in \widetilde{M}$ and any open neighborhood $V \subseteq \widetilde{M}$ of $q$, there exists an open neighborhood $W \subseteq V$ of $q$ and a constant $\beta>0$ such that $\left|\int_{x} \theta\right| \leq \beta l(x)^{2}$ for any closed curve $x: I \rightarrow W$. Proof of Proposition 5.3. Compactness of $M$ and the previous lemma imply that there exists $\beta, \rho_{0}>0$ such that if $x: I \rightarrow M$ is any closed contractible curve with $x(I)$ contained in a ball of radius $\rho_{0}$ then $\left|\int_{C(x)} \sigma\right| \leq \beta l(x)^{2}$. Let $q:=x_{0}(0)$ and let $W$ denote the ball of radius $\rho_{0}$ about $q$. Pick $\rho \in \mathbb{R}^{+}$such that

$$
0<\rho<\min \left\{\rho_{0}, \sqrt{k /(2 \beta)^{2}}\right\}
$$

Write $f(s)=\left(x_{s}, T_{s}\right)$, so $x_{s} \in \Lambda_{0}$ for all $s$. We claim that there exists $s_{0} \in(0,1)$ such that $l\left(x_{s_{0}}\right)=\rho$. Since the functional $s \mapsto l\left(x_{s}\right)$ is continuous and $l\left(x_{0}\right)=0$, it suffices to show that there exists $s_{1} \in[0,1)$ such that $l\left(x_{s_{1}}\right)>\rho$.

If there exists $s_{1} \in[0,1)$ such that $x_{s_{1}}(I) \varsubsetneqq W$, then we are done, since then $l\left(x_{s_{1}}\right) \geq \rho_{0}>\rho$. The other possibility is that $x_{s}(I) \subseteq W$ for all $s \in I$. In this case we claim that we may take $s_{1}=0$, that is, $l\left(x_{0}\right)>\rho$. By assumption if $y_{0}(t)=x_{0}\left(t / T_{0}\right)$, we have

$$
\begin{aligned}
0>S_{k}\left(x_{0}, T_{0}\right) & =\int_{0}^{1} \frac{1}{2 T_{0}}\left|\dot{x}_{0}(t)\right|^{2} d t+k T_{0}-\int_{C\left(x_{0}\right)} \sigma \\
& \geq \frac{1}{2 T_{0}} l\left(x_{0}\right)^{2}+k T_{0}-\left|\int_{x_{0}} \theta\right| \\
& \geq\left(\frac{1}{2 T_{0}}-\beta\right) l\left(x_{0}\right)^{2}+k T_{0},
\end{aligned}
$$

where the second inequality came from (3-1) and the third from Lemma 5.4. From this it follows that $T_{0}>1 /(2 \beta)$, and thus

$$
l\left(x_{0}\right)^{2}>\frac{k T_{0}}{\beta-1 /\left(2 T_{0}\right)}>\frac{k}{2 \beta^{2}}>\rho^{2} .
$$


and we are done as before.

We claim finally that $S_{k}\left(f\left(s_{0}\right)\right)>0$. Since $x_{s_{0}} \in C_{M}^{\mathrm{ac}}(q, q)$ and $l\left(x_{s_{0}}\right)<\rho_{0}$, we have $x_{s_{0}}(I) \subseteq W$. In particular, (5-2) holds for $x_{s_{0}}$, and so we have

$$
S_{k}\left(f\left(s_{0}\right)\right) \geq\left(\frac{1}{2 T_{s_{0}}}-\beta\right) \ell^{2}+k T_{s_{0}}=P\left(T_{s_{0}}\right) \geq \min _{t \in \mathbb{R}^{+}} P(t),
$$

where $P(t):=(1 /(2 t)-\beta) \rho^{2}+k t$. It is elementary to see that

$$
\min _{t \in \mathbb{R}^{+}} P(t)=\sqrt{\rho^{2} /(2 k)}=: \mu_{0}>0,
$$

and this completes the proof.

The next lemma will be needed to prove relative completeness of the flow of $-\nabla S_{k}$ on any interval not containing zero.

Lemma 5.5. There exists a constant $C>0$ such that for any $\left(x_{0}, T_{0}\right) \in \Lambda_{M} \times \mathbb{R}^{+}$ and any $r>0$, if $\left(x_{1}, T_{1}\right) \in \Lambda_{M} \times \mathbb{R}^{+}$satisfies $\operatorname{dist}\left(\left(x_{0}, T_{0}\right),\left(x_{1}, T_{1}\right)\right)<r$, then

$$
\left|T_{0}-T_{1}\right|<r \text { and } \operatorname{dist}_{\mathrm{HD}}\left(x_{0}, x_{1}\right)<C r \text {. }
$$

This result is essentially proved by Contreras [2006, Lemma 2.3]; Contreras used a different metric on $\Lambda_{M} \times \mathbb{R}^{+}$, which meant that an additional condition was imposed in the statement of the lemma. Since we are working with the standard metric (2-1) on $\Lambda_{M} \times \mathbb{R}^{+}$this additional condition is not needed, and the proof in [Contreras 2006] goes through without any changes.

Corollary 5.6. Let $K \subseteq \widetilde{M}$ and $B>0$. Let $\mathcal{U}:=\left\{(x, T) \in \Lambda_{0}^{K} \times \mathbb{R}^{+}: T \leq B\right\}$. Let $C$ be as in the statement of Lemma 5.5. Then if $L \subseteq \widetilde{M}$ satisfies

$$
\left\{q \in \tilde{M}: \operatorname{dist}_{\tilde{g}}\left(q, q^{\prime}\right) \leq C r \text { for some } q^{\prime} \in K\right\} \subseteq L
$$

and we set $\mathscr{V}:=\left\{(x, T) \in \Lambda_{0}^{L} \times \mathbb{R}^{+}: T \leq B+r\right\}$, then $\mathscr{B}_{r}(U) \subseteq \mathscr{V}$.

Proof. Suppose $\left(x_{1}, T_{1}\right) \in \mathscr{B}_{r}(U)$. Then there exists $\left(x_{0}, T_{0}\right) \in U$ with

$$
\operatorname{dist}\left(\left(x_{0}, T_{0}\right),\left(x_{1}, T_{1}\right)\right)<r .
$$

By Lemma 5.5, $\operatorname{dist}_{\mathrm{HD}}\left(x_{0}, x_{1}\right)<C r$ and $\left|T_{0}-T_{1}\right|<r$. Thus $\left(x_{1}, T_{1}\right) \in \mathscr{V}$.

Next, we prove relative completeness of the flow of $-\nabla S_{k}$ on any interval that doesn't contain zero. This proof is very similar to [Contreras 2006, Lemma 6.9].

Lemma 5.7. For all $k \in \mathbb{R}^{+}$, if $[a, b] \subseteq \mathbb{R}$ is an interval such that $0 \notin[a, b]$, then the local flow of $-\nabla S_{k}$ is relatively complete on $\left(\Lambda_{0} \times \mathbb{R}^{+}\right) \cap\left\{a \leq S_{k} \leq b\right\}$.

Proof. Let $\Phi_{s}: \Lambda_{M} \times \mathbb{R}^{+} \rightarrow \Lambda_{M} \times \mathbb{R}^{+}$denote the local flow of the vector field $-\nabla S_{k}$. Then for any $(x, T) \in \Lambda_{M} \times \mathbb{R}^{+}$,

$$
S_{k}\left(\Phi_{s_{1}}(x, T)\right)-S_{k}\left(\Phi_{s_{2}}(x, T)\right)=\int_{s_{1}}^{s_{2}}\left|\nabla S_{k}\left(\Phi_{s}(x, T)\right)\right|^{2} d s .
$$


By the Cauchy-Schwarz inequality we see that

$$
\begin{aligned}
\operatorname{dist}\left(\Phi_{s_{1}}(x, T), \Phi_{s_{2}}(x, T)\right)^{2} & \leq\left(\int_{s_{1}}^{s_{2}}\left|\nabla S_{k}\left(\Phi_{s}(x, T)\right)\right| d s\right)^{2} \\
& \leq\left|s_{1}-s_{2}\right| \int_{s_{1}}^{s_{2}}\left|\nabla S_{k}\left(\Phi_{s}(x, T)\right)\right|^{2} d s
\end{aligned}
$$

and hence

$$
\operatorname{dist}\left(\Phi_{s_{1}}(x, T), \Phi_{s_{2}}(x, T)\right)^{2} \leq\left|s_{1}-s_{2}\right|\left|S_{k}\left(\Phi_{s_{1}}(x, T)\right)-S_{k}\left(\Phi_{s_{2}}(x, T)\right)\right| .
$$

Now suppose we are given a pair $(x, T) \in \Lambda_{0} \times \mathbb{R}^{+}$, such that there exists $a, b \in \mathbb{R}$ with $0 \notin[a, b]$ and

$$
a \leq S_{k}\left(\Phi_{s}(x, T)\right) \leq b \text { for all } s \text { such that } \Phi_{s}(x, T) \text { is defined. }
$$

Let $[0, \alpha)$ be the maximum interval of definition of $s \mapsto \Phi_{s}(x, T)$, where $\alpha>0$. To complete the proof we need to show $\alpha=\infty$. Suppose the contrary.

Write $\Phi_{s}(x, T)=\left(x_{s}, T_{s}\right)$. If $s_{n} \uparrow \alpha$, then $\left(\Phi_{s_{n}}(x, T)\right)=:\left(x_{n}, T_{n}\right)$ is a Cauchy sequence in $\left(\Lambda_{0} \times \mathbb{R}^{+}\right) \cap\left\{a \leq S_{k} \leq b\right\}$ by (5-3). Thus $T_{\alpha}:=\lim _{s \uparrow \alpha} T_{s}$ exists and is finite.

If $T_{\alpha}>0$, then $\left(x_{\alpha}, T_{\alpha}\right):=\lim _{n \rightarrow \infty}\left(x_{n}, T_{n}\right)$ exists and is equal to $\Phi_{\alpha}(x, T)$ since the sequence $\left(x_{n}, T_{n}\right)$ is Cauchy. Since $S_{k}$ is $C^{2}$ we can extend the solution $s \mapsto \Phi_{s}(x, T)$ at $s=\alpha$, contradicting the definition of $\alpha$. Thus $T_{\alpha}=0$. Hence there exists a sequence $s_{m} \uparrow \alpha$ such that $\frac{d}{d s} T_{s_{m}} \leq 0$. As before write $x_{m}:=x_{s_{m}}$ and $T_{m}:=T_{s_{m}}$. By (5-3) and Lemma 5.5, we may assume there exists a compact set $K \subseteq \tilde{M}$ such that $\left(x_{m}, T_{m}\right) \subseteq \Lambda_{0}^{K} \times \mathbb{R}^{+}$for all $m$. If $y_{m}(t):=x_{m}\left(t / T_{m}\right)$, then

$$
0 \geq \frac{d}{d s} T_{m}=-\frac{\partial}{\partial T} S_{k}\left(x_{m}, T_{m}\right)=\frac{1}{T_{m}} \int_{0}^{T_{m}}\left(-k+E\left(y_{m}, \dot{y}_{m}\right)\right) d t=-k+\frac{e_{m}}{T_{m}},
$$

where the penultimate equality uses (2-6). Since $\lim _{m \rightarrow \infty} T_{m}=0$, this forces $\lim _{m \rightarrow \infty} e_{m}=0$. As in the proof of the second part of Theorem 3.2, this implies $S_{k}\left(x_{m}, T_{m}\right) \rightarrow 0$, contradicting the fact that $0 \notin[a, b]$. This implies that we must have originally had $\alpha=\infty$, and so completes the proof.

We now move towards proving Theorem 1.1(2). In fact, we will prove a stronger result, which is based on [Contreras 2006, Proposition 7.1]:

Proposition 5.8. Let $c=c(g, \sigma) \in \mathbb{R} \cup\{\infty\}$. For almost all $k \in(0, c)$ there exists $a$ contractible closed orbit of $\phi_{t}$ with energy $k$. This orbit has positive $S_{k}$-action, and is not a strict local minimizer of $S_{k}$ on $\Lambda_{0} \times \mathbb{R}^{+}$. This holds for a specific $k \in(0, c)$ if $S_{k}$ is known to satisfy the Palais-Smale condition on the level $k$.

Proof. Fix $k_{0} \in(0, c)$. There exists $\left(x_{0}, T_{0}\right) \in \Lambda_{0} \times \mathbb{R}^{+}$such that $S_{k_{0}}\left(x_{0}, T_{0}\right)<0$. Indeed, there exists a closed curve $\tilde{y}:\left[0, T_{0}\right] \rightarrow \widetilde{M}$ such that $A_{k_{0}}(\tilde{y})<0$. Then the 
projection $y:\left[0, T_{0}\right] \rightarrow M$ of $\tilde{y}$ to $M$ is a closed curve, and if $x_{0}(t):=y\left(t T_{0}\right)$, then $\left(x_{0}, T_{0}\right) \in \Lambda_{0} \times \mathbb{R}^{+}$and $S_{k_{0}}\left(x_{0}, T_{0}\right)=A_{k_{0}}(\tilde{y})<0$. There exists $\varepsilon>0$ such that for all $k \in J:=\left[k_{0}, k_{0}+\varepsilon\right]$, we have $S_{k}\left(x_{0}, T_{0}\right)<0$.

Let $x_{1}$ denote the constant loop at $x_{0}(0)$. Given $k \in J$, let $\mu_{0}(k)>0$ be the constant given by Proposition 5.3 such that any path $f \in C^{0}\left(I, \Lambda_{0} \times \mathbb{R}^{+}\right)$with $f(0)=\left(x_{0}, T_{0}\right)$ and $f(1)=\left(x_{1}, T\right)$ for some $T>0$ has $\sup _{s \in I} S_{k}(f(s))>\mu_{0}(k)$. Choose $T_{1}>0$ such that $T_{1}<\inf _{k \in J} \mu_{0}(k) / k$. Then

$$
\max \left\{S_{k}\left(x_{0}, T_{0}\right), S_{k}\left(x_{1}, T_{1}\right)\right\}=k T_{1}<\mu_{0}(k) \text { for all } k \in J .
$$

Set $\Gamma:=\left\{f \in C^{0}\left(I, \Lambda_{0} \times \mathbb{R}^{+}\right): f(0)=\left(x_{0}, T_{0}\right), f(1)=\left(x_{1}, T_{1}\right)\right\}$. Let $\left(K_{n}\right) \subseteq \widetilde{M}$ denote compact sets such that $K_{n} \subseteq K_{n+1}$ and $\bigcup_{n} K_{n}=\tilde{M}$. Let

$$
\Gamma_{n}:=\Gamma \cap C^{0}\left(I, \Lambda_{0}^{K_{n}} \times \mathbb{R}^{+}\right) .
$$

Define $\mu_{n}(k):=\inf _{f \in \Gamma_{n}} \sup _{s \in I} S_{k}(f(s))$ and $\mu_{\infty}(k):=\inf _{f \in \Gamma} \sup _{s \in I} S_{k}(f(s))$ for $k \in J$. Then $\mu_{n}(k) \geq \mu_{n+1}(k) \geq \mu_{\infty}(k) \geq \mu_{0}(k)$ for all $n \in \mathbb{N}$ and $k \in J$, and the functions $\mu_{n}: J \rightarrow \mathbb{R}$ converge pointwise to $\mu_{\infty}$. Both $\mu_{n}$ and $\mu_{\infty}$ are nondecreasing. Since $\mu_{\infty}$ is nondecreasing, by Lebesgue's theorem there exists a subset $J_{0} \subseteq\left(k_{0}, k_{0}+\varepsilon\right)$ with $J \backslash J_{0}$ having measure zero such that $\left.\mu_{\infty}\right|_{J_{0}}$ is locally Lipschitz. In other words, for all $j \in J_{0}$ there exist constants $M(j)>0$ and $\delta(j)>0$ such that

$$
\left|\mu_{\infty}(j+\delta)-\mu_{\infty}(j)\right|<M(j)|\delta| \text { for all }|\delta|<\delta(j) .
$$

Fix $j \in J_{0}$ and a sequence $\left(j_{m}\right) \subseteq J_{0}$ with $j_{m} \downarrow j$. Let $f_{n, m} \in \Gamma_{n}$ be paths such that

$$
\max _{s \in I} S_{j_{m}}\left(f_{n, m}(s)\right) \leq \mu_{n}\left(j_{m}\right)+\left(j_{m}-j\right) .
$$

Next, define $\mathcal{U}_{n}:=\left\{(x, T) \in \Lambda_{0}^{K_{n}} \times \mathbb{R}^{+}: T \leq M(j)+2\right\}$. Choose another collection $\left(L_{n}\right) \subseteq \tilde{M}$ of compact sets such that $K_{n} \subseteq L_{n}$, and such that $\mathscr{B}_{1}\left(\mathcal{U}_{n}\right) \subseteq \mathscr{V}_{n}$ for $\mathscr{V}_{n}:=\left\{(x, T) \in \Lambda_{0}^{L_{n}} \times \mathbb{R}^{+}: T \leq M(j)+3\right\}$. Such a collection $\left(L_{n}\right)$ exists by Corollary 5.6. Since $\mu_{\infty}(j) \neq 0$, from Proposition 3.7 it follows that $\left.S_{j}\right|_{q_{n}}$ satisfies the Palais-Smale condition at the level $\mu_{\infty}(j)$ for all $n \in \mathbb{N}$.

Since $k \mapsto S_{k}(x, T)$ is increasing,

$$
\max _{s \in I} S_{j}\left(f_{n, m}(s)\right) \leq \max _{s \in I} S_{j_{m}}\left(f_{n, m}(s)\right) \leq \mu_{n}\left(j_{m}\right)+\left(j_{m}-j\right) .
$$

If $s \in I$ is such that $S_{j}\left(f_{n, m}(s)\right)>\mu_{\infty}(j)-\left(j_{m}-j\right)$, writing $f_{n, m}(s)=:\left(x_{s}^{n, m}, T_{s}^{n, m}\right)$ we have

$$
\begin{aligned}
T_{s}^{n, m} & =\frac{S_{j_{m}}\left(f_{n, m}(s)\right)-S_{j}\left(f_{n, m}(s)\right)}{j_{m}-j} \\
& \leq \frac{\mu_{\infty}\left(j_{m}\right)-\mu_{n}(j)}{j_{m}-j}+2 \leq \frac{\mu_{\infty}\left(j_{m}\right)-\mu_{\infty}(j)}{j_{m}-j}+2 \leq M(j)+2,
\end{aligned}
$$


for $n$ large enough.

Given $\varepsilon>0$, first choose $m$ large enough that $j_{m}-j<\varepsilon /(2(M(j)+1))$, and then select $n$ large enough that $\mu_{n}\left(j_{m}\right)-\mu_{\infty}\left(j_{m}\right)<\varepsilon / 2$. Then

$$
\begin{aligned}
\mu_{n}\left(j_{m}\right)- & \mu_{\infty}(j)+\left(j_{m}-j\right) \\
& =\left(\mu_{n}\left(j_{m}\right)-\mu_{\infty}\left(j_{m}\right)\right)+\left(\mu_{\infty}\left(j_{m}\right)-\mu_{\infty}(j)\right)+\left(j_{m}-j\right) \\
& <\varepsilon / 2+M(j)\left(j_{m}-j\right)+\left(j_{m}-j\right)<\varepsilon .
\end{aligned}
$$

Then by (5-4),

$$
f_{n, m}(I) \subseteq\left\{S_{j} \leq \mu_{\infty}(j)-\left(j_{m}-j\right)\right\} \cap\left(u_{n} \cap\left\{S_{j} \leq \mu_{\infty}(j)+\varepsilon\right\}\right) .
$$

Since $\mu_{\infty}(j) \neq 0$, by Lemma 5.7 the gradient flow of $-S_{j}$ is relatively complete on $\left\{\mu_{\infty}(j)-\eta \leq S_{j} \leq \mu_{\infty}(j)+\eta\right\}$ for some $\eta>0$. Theorem 5.1 then gives a critical point for $\left.S_{j}\right|_{\Lambda_{0} \times \mathbb{R}^{+}}$that is not a strict local minimizer (here we are applying Theorem 5.1 with $\left.\mathscr{F}_{n}:=\left\{f(I): f \in \Gamma_{n}\right\}\right)$.

Finally, suppose that $k<c(g, \sigma) \leq \infty$ is such that $S_{k}$ satisfies the Palais-Smale condition. Then the theorem is immediate from Lemma 5.7 and Theorem 5.1. Indeed, by Lemma 5.7 we may simply take $U_{n}=\mathscr{V}_{n}=\mathscr{V}_{\infty}=\Lambda_{0} \times \mathbb{R}^{+}$, as then the hypotheses of Theorem 5.1 are trivially satisfied. This completes the proof of Theorem 1.1.

\section{Acknowledgment}

I thank my PhD adviser Gabriel P. Paternain for many helpful discussions.

\section{References}

[Abbondandolo and Schwarz 2009a] A. Abbondandolo and M. Schwarz, "Estimates and computations in Rabinowitz-Floer homology”, J. Topol. Anal. 1:4 (2009), 307-405. MR 2597650

[Abbondandolo and Schwarz 2009b] A. Abbondandolo and M. Schwarz, "A smooth pseudo-gradient for the Lagrangian action functional”, Adv. Nonlinear Stud. 9:4 (2009), 597-623. MR 2560122 Zbl 1185.37145

[Burns and Paternain 2002] K. Burns and G. P. Paternain, "Anosov magnetic flows, critical values and topological entropy”, Nonlinearity 15:2 (2002), 281-314. MR 2004d:37076 Zbl 1161.37337

[Cieliebak et al. 2010] K. Cieliebak, U. Frauenfelder, and A. Oancea, "Rabinowitz Floer homology and symplectic homology”, Annales scientifiques de l'ENS 43 (2010), fascicule 6. arXiv 0903.0768

[Contreras 2006] G. Contreras, "The Palais-Smale condition on contact type energy levels for convex Lagrangian systems”, Calc. Var. Partial Differential Equations 27:3 (2006), 321-395. MR 2007i:37116 Zbl 1105.37037

[Contreras and Iturriaga 1999] G. Contreras and R. Iturriaga, Global minimizers of autonomous Lagrangians, Colóquio Brasileiro de Matemática 22, Instituto de Matemática Pura e Aplicada, Rio de Janeiro, 1999. MR 2001j:37113 Zbl 0957.37065 
[Contreras et al. 1997] G. Contreras, J. Delgado, and R. Iturriaga, "Lagrangian flows: The dynamics of globally minimizing orbits, II”, Bol. Soc. Brasil. Mat. (N.S.) 28:2 (1997), 155-196. MR 98i:58093 Zbl 0892.58065

[Contreras et al. 2000] G. Contreras, R. Iturriaga, G. P. Paternain, and M. Paternain, "The PalaisSmale condition and Mañé's critical values", Ann. Henri Poincaré 1:4 (2000), 655-684. MR 2001k: $37101 \mathrm{Zbl} 0986.58005$

[Contreras et al. 2004] G. Contreras, L. Macarini, and G. P. Paternain, "Periodic orbits for exact magnetic flows on surfaces", Int. Math. Res. Not. 2004:8 (2004), 361-387. MR 2005a:37103 Zbl 1086.37032

[Ginzburg 1996] V. L. Ginzburg, "On closed trajectories of a charge in a magnetic field: An application of symplectic geometry", pp. 131-148 in Contact and symplectic geometry (Cambridge, 1994), edited by C. B. Thomas, Publ. Newton Inst. 8, Cambridge Univ. Press, 1996. MR 97j:58128 Zbl 0873.58034

[Ginzburg and Gürel 2009] V. L. Ginzburg and B. Z. Gürel, "Periodic orbits of twisted geodesic flows and the Weinstein-Moser theorem", Comment. Math. Helv. 84 (2009), 865-907. MR 2534483 Zbl 1184.37046

[Kerman 2000] E. Kerman, Symplectic geometry and the motion of a particle in a magnetic field, thesis, Univ. California, Santa Cruz, 2000, available at http://tinyurl.com/32h3bzu. MR 2616826

[Lu 2006] G. Lu, "Finiteness of the Hofer-Zehnder capacity of neighborhoods of symplectic submanifolds", Int. Math. Res. Not. 2006 (2006), Art. ID 76520. MR 2007a:53159 Zbl 1132.53317

[Mañé 1996] R. Mañé, "Lagrangian flows: The dynamics of globally minimizing orbits”, pp. 120131 in International Conference on Dynamical Systems (Montevideo, 1995), edited by F. Ledrappier et al., Pitman Res. Notes Math. Ser. 362, Longman, Harlow, 1996. MR 98g:58059 Zbl 0870. 58026

[Macarini 2004] L. Macarini, "Hofer-Zehnder capacity and Hamiltonian circle actions", Commun. Contemp. Math. 6:6 (2004), 913-945. MR 2005k:53170 Zbl 1076.53098

[Merry 2010] W. J. Merry, "On the Rabinowitz Floer homology of twisted cotangent bundles", preprint, 2010. arXiv 1002.0162

[Osuna 2005] O. Osuna, "Periodic orbits of weakly exact magnetic flows", preprint, 2005.

[Paternain 2006] G. P. Paternain, "Magnetic rigidity of horocycle flows", Pacific J. Math. 225:2 (2006), 301-323. MR 2008e:37029 Zbl 1116.37020

[Polterovich 1998] L. Polterovich, "Geometry on the group of Hamiltonian diffeomorphisms", pp. 401-410 in Proceedings of the International Congress of Mathematicians (Berlin, 1998), vol. 2, Doc. Math. 1998, 1998. MR 2000c:37120 Zbl 0909.58004

[Schlenk 2006] F. Schlenk, "Applications of Hofer's geometry to Hamiltonian dynamics", Comment. Math. Helv. 81:1 (2006), 105-121. MR 2007f:53117 Zbl 1094.37031

[Usher 2009] M. Usher, "Floer homology in disk bundles and symplectically twisted geodesic flows", J. Mod. Dyn. 3:1 (2009), 61-101. MR 2010b:53157 Zbl 1186.53099

Received June 28, 2009.

\section{WILL J. MERRY}

Department of Pure Mathematics and Mathematical Statistics

UNIVERSITY OF CAMBRIDGE

\section{CAMBRIDGE CB3 0WB}

ENGLAND

w.merry@dpmms.cam.ac.uk 


\title{
PACIFIC JOURNAL OF MATHEMATICS
}

\author{
http://www.pjmath.org \\ Founded in 1951 by \\ E. F. Beckenbach (1906-1982) and F. Wolf (1904-1989)
}

\section{EDITORS}

V. S. Varadarajan (Managing Editor)

Department of Mathematics

University of California

Los Angeles, CA 90095-1555

pacific@math.ucla.edu

Vyjayanthi Chari

Department of Mathematics

University of California

Riverside, CA 92521-0135

chari@math.ucr.edu

Robert Finn

Department of Mathematics Stanford University

Stanford, CA 94305-2125

finn@math.stanford.edu

Kefeng Liu

Department of Mathematics

University of California

Los Angeles, CA 90095-1555

liu@math.ucla.edu
Darren Long

Department of Mathematics

University of California

Santa Barbara, CA 93106-3080

long@math.ucsb.edu

Jiang-Hua Lu

Department of Mathematics

The University of Hong Kong

Pokfulam Rd., Hong Kong jhlu@maths.hku.hk

Alexander Merkurjev

Department of Mathematics

University of California

Los Angeles, CA 90095-1555

merkurev@math.ucla.edu
Sorin Popa

Department of Mathematics University of California

Los Angeles, CA 90095-1555 popa@math.ucla.edu

Jie Qing

Department of Mathematics

University of California

Santa Cruz, CA 95064

qing@cats.ucsc.edu

Jonathan Rogawski

Department of Mathematics

University of California

Los Angeles, CA 90095-1555

jonr@math.ucla.edu

\section{PRODUCTION}

pacific@math.berkeley.edu

\begin{abstract}
Silvio Levy, Scientific Editor Matthew Cargo, Senior Production Editor
\end{abstract}
ACADEMIA SINICA, TAIPEI

CALIFORNIA INST. OF TECHNOLOGY

INST. DE MATEMÁTICA PURA E APLICADA

KEIO UNIVERSITY

MATH. SCIENCES RESEARCH INSTITUTE

NEW MEXICO STATE UNIV.

OREGON STATE UNIV.

\section{SUPPORTING INSTITUTIONS}

STANFORD UNIVERSITY
UNIV. OF BRITISH COLUMBIA
UNIV. OF CALIFORNIA, BERKELEY
UNIV. OF CALIFORNIA, DAVIS
UNIV. OF CALIFORNIA, LOS ANGELES
UNIV. OF CALIFORNIA, RIVERSIDE
UNIV. OF CALIFORNIA, SAN DIEGO
UNIV. OF CALIF., SANTA BARBARA

UNIV. OF CALIF., SANTA CRUZ

UNIV. OF MONTANA

UNIV. OF OREGON

UNIV. OF SOUTHERN CALIFORNIA

UNIV. OF UTAH

UNIV. OF WASHINGTON

WASHINGTON STATE UNIVERSITY

These supporting institutions contribute to the cost of publication of this Journal, but they are not owners or publishers and have no responsibility for its contents or policies.

See inside back cover or www.pjmath.org for submission instructions.

The subscription price for 2010 is US \$420/year for the electronic version, and \$485/year for print and electronic.

Subscriptions, requests for back issues from the last three years and changes of subscribers address should be sent to Pacific Journal of Mathematics, P.O. Box 4163, Berkeley, CA 94704-0163, U.S.A. Prior back issues are obtainable from Periodicals Service Company, 11 Main Street, Germantown, NY 12526-5635. The Pacific Journal of Mathematics is indexed by Mathematical Reviews, Zentralblatt MATH, PASCAL CNRS Index, Referativnyi Zhurnal, Current Mathematical Publications and the Science Citation Index.

The Pacific Journal of Mathematics (ISSN 0030-8730) at the University of California, c/o Department of Mathematics, 969 Evans Hall, Berkeley, CA 94720-3840, is published monthly except July and August. Periodical rate postage paid at Berkeley, CA 94704, and additional mailing offices. POSTMASTER: send address changes to Pacific Journal of Mathematics, P.O. Box 4163, Berkeley, CA 94704-0163.

PJM peer review and production are managed by EditFLOW ${ }^{\mathrm{TM}}$ from Mathematical Sciences Publishers.

PUBLISHED BY PACIFIC JOURNAL OF MATHEMATICS

at the University of California, Berkeley 94720-3840

A NON-PROFIT CORPORATION

Typeset in LATEX

Copyright $(\mathrm{C} 2010$ by Pacific Journal of Mathematics 


\section{PACIFIC JOURNAL OF MATHEMATICS}

Volume $247 \quad$ No. $1 \quad$ September 2010

Classification results for easy quantum groups

TEOdor BANiCA, StePhen CURRAN and Roland SPEICHER

Batalin-Vilkovisky coalgebra of string topology

XiAOJUn CHEN and WeE LiAnG GAN

Invariant Finsler metrics on polar homogeneous spaces

SHAOQIANG DENG

A proof of the Concus-Finn conjecture

KIRK E. LANCASTER

The existence and monotonicity of a three-dimensional transonic shock in a finite nozzle with axisymmetric exit pressure

JUn Li, ZHOUPING XIN and HUICHENG YiN

Bi-Hamiltonian flows and their realizations as curves in real semisimple homogeneous manifolds

GLORIA MARÍ BEFFA

Closed orbits of a charge in a weakly exact magnetic field

WILL J. MERRY

Ringel-Hall algebras and two-parameter quantized enveloping algebras

XIN TANG

A new probability distribution with applications

MINGJIN WANG 